\title{
MAC PROTOCOL ADAPTATION IN COGNITIVE RADIO NETWORKS
}

\author{
by \\ KUO-CHUN HUANG
}

\author{
A thesis submitted to the \\ Graduate School-New Brunswick \\ Rutgers, The State University of New Jersey \\ in partial fulfillment of the requirements \\ for the degree of \\ Master of Science \\ Graduate Program in Electrical and Computer Engineering \\ Written under the direction of \\ Professor Dipankar Raychaudhuri \\ and approved by
}

New Brunswick, New Jersey

October, 2010 


\title{
ABSTRACT OF THE THESIS \\ MAC PROTOCOL ADAPTATION IN COGNITIVE RADIO NETWORKS \\ By KUO-CHUN HUANG
}

\author{
Thesis Director: \\ Professor Dipankar Raychaudhuri
}

This thesis presents an adaptive MAC (AMAC) protocol for supporting MAC layer adaptation in cognitive radio networks. MAC protocol adaptation is motivated by the flexibility of emerging software-defined radios which make it feasible to dynamically adjust radio protocols and parameters in order to maintain communications quality. Dynamic changes to the MAC layer may be useful in tactical or vehicular networking scenarios, where radio node density, traffic volumes and service requirements can vary widely over time. A specific control framework for the proposed AMAC algorithm is described based on the "CogNet" protocol stack which uses a Global Control Plane (GCP) to distribute control information between nearby radios. An AMAC prototype which switches between CSMA and TDMA is evaluated for various traffic scenarios using the NS-2 simulator. In addition, a proof-of-concept AMAC is implemented using GNUradio/USRP platforms on the ORBIT radio grid testbed. Detailed simulation and experimental results are given for both UDP and TCP traffic with different usage scenarios and application models. The results show that AMAC can provide improved performance relative to a conventional static system and can be implemented with reasonable control protocol overhead and latency. 


\section{Table of Contents}

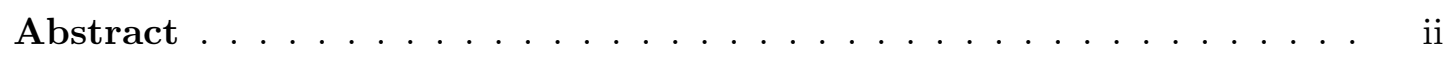

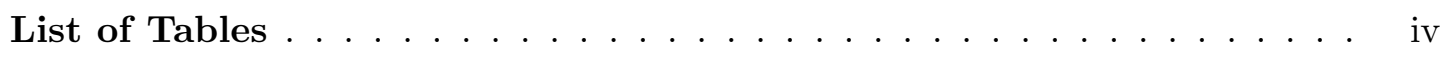

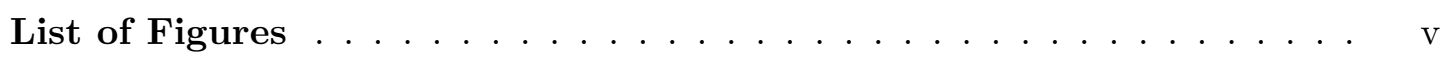

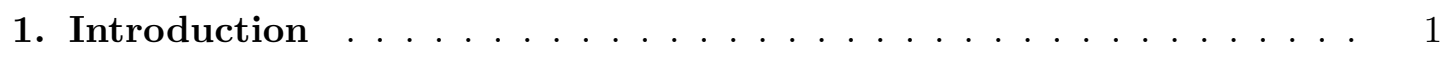

2. Related Work ...................... 5

3. Network Architecture . . . . . . . . . . . . . . 7

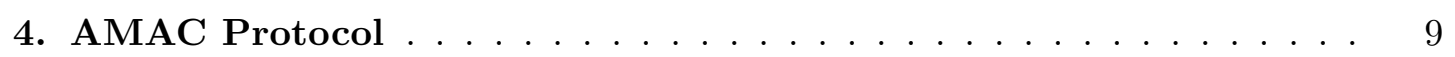

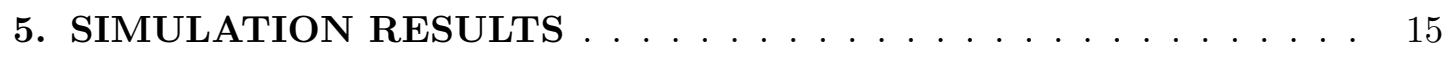

5.1. Scenario with Mixed Traffic Pattern . . . . . . . . . . . . . 16

5.2. Scenario with Mobile Environment (network type) . . . . . . . . . 18

5.3. Scenario with Mobile Environment (service requirement) . . . . . . . . 20

6. EXPERIMENTAL EVALUATION ON GNU RADIO/ORBIT . . . 24

6.1. Experiment Setup . . . . . . . . . . . . . . . . 24

6.2. Experimental Results . . . . . . . . . . . . . . . . 26

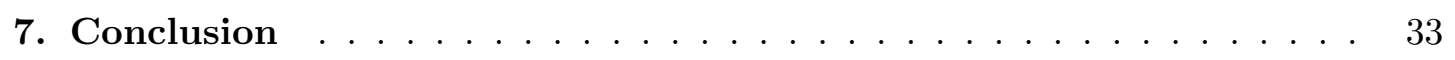

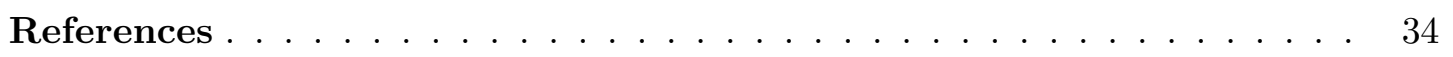

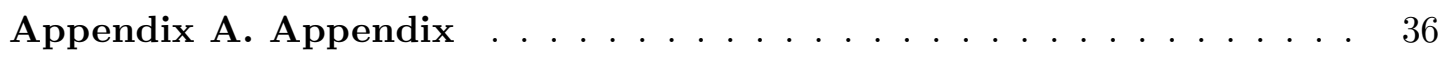




\section{List of Tables}

4.1. GCP beacon message format . . . . . . . . . . . . . . . 10

4.2. Control message format for MAC switching request . . . . . . . . . . . . 12

5.1. GENERAL SIMULATION PARAMETERS . . . . . . . . . . . . . . . 15

6.1. GNURADIO(Data Plane) radio parameters . . . . . . . . . . . . 25

6.2. AMAC overhead from GCP control traffic . . . . . . . . . . . . . . . 32 


\section{List of Figures}

3.1. GCP and Data Plane structure . . . . . . . . . . . . 8

4.1. Example network of AMAC protocol . . . . . . . . . . . . . . . . . . . 14

5.1. Characteristics of CSMA and TDMA with streaming traffic . . . . . . 17

5.2. Characteristics of CSMA and TDMA with bursty traffic . . . . . . . 18

5.3. Flow traffic type in mix traffic scenario . . . . . . . . . . . . . . . 19

5.4. Average throughput of mix traffic scenario . . . . . . . . . . . . 20

5.5. Mobile scenario with different MAC-type networks . . . . . . . . . . 21

5.6. Average network throughput of city, mobile and suburban networks . . 22

5.7. Mobile scenario with mix service types . . . . . . . . . . . . . . . 22

5.8. Average network throughput of VoIP, streaming and web browsing net-

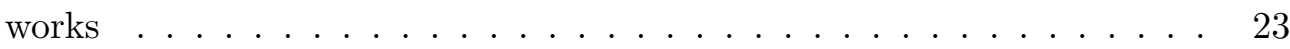

6.1. Experimental network topology for AMAC . . . . . . . . . . . 25

6.2. Average throughput of UDP traffic with larger packet size . . . . . . . . 27

6.3. Average throughput of UDP traffic with smaller packet size . . . . . . 27

6.4. Average throughput of UDP traffic with smaller packet size . . . . . 28

6.5. Average throughput of CSMA, TDMA and AMAC over TCP transmission 29

6.6. Topology of multi-hop scenario . . . . . . . . . . . . . . . . . . 30

6.7. Throughput comparison varied with time between CSMA, TDMA and

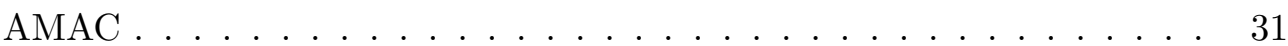

6.8. Average throughput of CSMA, TDMA and AMAC . . . . . . . . . 31

A.1. NS-2 programming flow chart . . . . . . . . . . . . 36

A.2. GNUradio programming stacks . . . . . . . . . . . . . 37 


\section{Chapter 1}

\section{Introduction}

The concept of cognitive radio (CR) [1] is to dynamically choose a wide variety of radio parameters and network protocol standards in order to adapt to observed radio link and network conditions. Such a cognitive radio system is capable of handling crosslayer parameter changes: at the physical layer, the agile radio can search so-called

spectrum white space from unlicensed and even licensed bands to make good use of the spectrum opportunities with power control and modulation waveform adaptation; at the network layer, the cognitive radio system can also make runtime changes to the protocols used for packet transmission, to avoid collisions and congestion when network topology or traffic characteristics change. The ability to adapt different protocol layers with cognitive radio technology offers the prospect of improved networking performance in dynamic wireless networking scenarios such as tactical and vehicular.

There have been many research works addressing the physical layer agility of a cognitive radio system based on dynamic spectrum access (DSA) technique [2] [3]. However, there still is lack of a systematic study of how the cognitive radio performs with medium access control (MAC) protocol adaptation. This thesis proposes a novel adaptive MAC (AMAC) design for cognitive radio networks, with the ability to adapt between different types of MAC protocols based on the observation of network traffic changes. The candidate medium access protocols usually have different advantages in various network situations, and the AMAC protocol provides a framework to observe the runtime network changes and makes judgments for switching between those candidate protocols on the fly. 
The proposed AMAC protocol is based on the CogNet cognitive radio protocol architecture described in [4]. The CogNet architecture includes the concept of a "global control plane (GCP)" which supports exchange of control information between networking cognitive radio devices. MAC adaptation under consideration here requires exchange of local cross-layer parameters and tokens to maintain the protocol consistency between radio nodes sharing the same physical channel. The same GCP framework has been used in earlier work for dynamic spectrum coordination in multi-radio environments [5], as well as for cross-layer ad hoc network routing [4].

In this thesis, we focus on how to switch between different MAC protocols. Since the MAC protocols have different edges in different network scenarios, we propose three strategies for local nodes to determine what is the right time to initiate a switch. However, if different MAC protocols are used in the local network, different packet transmission schedules may cause more collisions than single MAC in the network's point of view. Thus, we choose a simple approach to derive the common MAC protocol for the smaller network. Additionally, in order to maintain MAC protocol consistency in the larger network, we introduce another approach of cross-layer design to reach common MAC protocol for the end-to-end link.

The candidate medium access control protocols we have chosen for evaluation represent the two most commonly used classes of wireless network protocols: CSMA and TDMA. CSMA is easy to implement and is efficient for supporting short packet transmissions from bursty sources with light to medium traffic volumes. In heavier traffic scenario, particularly with streaming sources, it is well known that TDMA provides a more efficient and reliable solution. In mobile environment, when the mobiles in data transmission are driving from city to suburban area, they can adapt MAC protocol from TDMA-based MAC to CSMA-based MAC. TDMA is able to reduce collision under dense network such as city area and CSMA provides flexibility under scattered network such as suburban area. A cell phone user, for instance, can use TDMA-based MAC for GSM system (voice transmission) and CSMA-based MAC for WiFi system (data transmission). Other MAC protocols can also be used in conjunction with AMAC, even though our GNU radio implementation is currently limited to CSMA and TDMA. 
We present the simulation and implementation aspects of AMAC protocol. NS2 simulator is used to emulate the behavior of AMAC in varying environments and network types. GNU radio on the ORBIT radio grid testbed [6] is the platform we use to validate AMAC's practicality. GNU radio is a type of software defined radio (SDR), which provides considerable flexibility for runtime adaptation of both physical layer signal parameters as well as MAC protocol functions. It integrates simple hardware and high functional software to construct simple radios which support intelligent control in cognitive radio network. There are two main components in GNUradio: host processor and USRP. USRP is a software radio transceiver that converts between digital and analog signals. It is able to support multiple radio band such as ISM band or $400 \mathrm{MHz}$ band. The host is a general-purpose computer that processes the baseband digitized raw signals and connects to USRP through USB cable. The Python/C++ based PHY modules are built in the GNUradio library and we can program under python interface to control PHY or radio parameters on-the-fly. However, due to the unpredictable processing delay in the host and high communication latency through USB, the MAC operations required timely responses are not supported yet. In this implementation, we build our own coarse TDMA MAC and basic CSMA MAC modules to validate GCP architecture and AMAC algorithm are implementable in the real world.

We evaluate the proposed AMAC protocol with various traffic types and environments. The purpose of the study is to validate the control protocol by which AMAC coordinates MAC protocol switching in different applications and measure the control overhead. In simulation, we compare CSMA and TDMA in bursty and streaming traffic scenarios and validate AMAC significantly improves throughput performance in dynamic traffic scenarios. In mobile network, we show the average throughput of mobile nodes transmission is improved by adapting different MAC protocols in different node density environments. For different service type networks, AMAC is shown to maintain service requirement and provides fairness for the neighbor networks. In implementation, we first present AMAC performance with baseline static TCP and UDP traffic and environment. Additionally, system performance in terms of throughput with varying UDP packet size is studied in the multihop scenario. These proof-of-concept 
GNU radio experiments within the ORBIT radio grid testbed at Rutgers University [6] are intended to demonstrate the feasibility of building an adaptive wireless network using the CogNet GCP architecture.

In the next chapter, we begin by describing related work. That is followed by our network architecture in chapter 3, the structure that we used for AMAC protocol. Then chapter 4 provides a detailed discussion of the proposed AMAC protocol and the associated adaptation algorithms. In chapter 5, several NS-2 simulation scenarios and performance results are presented. We provide experimental GNUradio prototyping results to further validate AMAC performance in chapter 6 and the conclusion is in chapter 7 . 


\section{Chapter 2}

\section{Related Work}

The benefits of separate control and data planes in the CogNet architecture have been previously explained in [4]. In [7], the Ad hoc network also uses separate control channel to transmit RTS/CTS control packets and selects the best data channel with highest SINR (signal-to-interference and noise ratio). The free data channel list is embedded in the RTS/CTS packet to find the best matching data channel. Our global control plane (GCP) uses a low-rate radio PHY with wide coverage for robustness, and has more functions which include protocol modules for topology discovery, bootstrapping, and address assignment. The data plane protocol stack supports data communication via PHY, MAC and routing modules specified through an API that interacts with the control modules in the radio node. This GCP control architecture can be used for various design objectives ranging from spectrum etiquette [8], adaptive MAC under consideration here, as well as cross-layer path setup and routing [4]. With the concept of fixed and separate control channel, this GCP architecture can maximize the use of spectrum without knowing the signal parameters in advance.

In [9], the author uses the rendezvous channel (RC) to coordinate among wireless nodes in different channels. C-MAC operates over decentralized multi-channels CR networks with dynamic RC. Also, each data superframe has to sacrifice its beacon period to get control information which causes overhead to affect data transmitting. In [10] existing MAC protocols are combined into a single higher layer, using only local network feedback information to determine the optimum MAC protocol. However, this work does not consider the global network view for switching different MACs and takes an amount of time to determine the optimum MAC after comparing the performance of all candidate MACs. 
In [11], a TDMA implementation for software defined radios is proposed. Different software frameworks for TDMA are used for implementation: GNU radio for PHY layer development and Click for protocol development. However, the time slot for this TDMA design is around 1 second, too large a value to be useful for most applications. Ref [12] modifies the functions required for faster processing by the CRs FPGA instead of the host CPU, providing for better timing in a MAC layer implementation. Ref [13] also uses GNU radios for PHY implementation and Click for MAC development and reports preliminary experiments in the Hydra system. Ref [14] proposes the hardware platform, KUAR, to develop the cognitive radio and also incorporate the SDR platforms to control the parameters. 


\section{Chapter 3}

\section{Network Architecture}

The proposed GCP-based cognitive radio network architecture is shown in Figure 3.1. A global control plane is used to carry all the control information exchanged between nodes while the data plane is dedicated for data transmission. The concept of GCP can be implemented using various methods such as a separate control radio or a control mode the agile radio can periodically switch to. In this thesis, we utilize an additional low-cost control radio operating on a dedicated control channel which is in charge of the resource reservation and allocation of radio and protocol parameters. The control radio is designed to have greater range and lower data rate as compared to the radio in data plane. The GCP offers basic control functions such as the initial bootstrapping as well as topology discovery when the new nodes join the network, and subsequently supports adaptive MAC operation when network or environment condition changes. Radio parameters could be optimized for end-to-end data transmission in different scenarios with different traffic situations.

The data plane protocol stack on each node, linked with the GCP through APIs, handles service data between the wireless nodes, while the GCP assists in establishing the operating PHY, MAC and routing parameters. Data is transmitted in the established data path negotiated using the GCP protocols. The actual data plane media access control protocols can be dynamically switched between candidate protocols in response to observed changes in network condition and/or service requirements. The AMAC algorithm is implemented in the control plane and candidate MAC protocols are implemented in the data plane. 


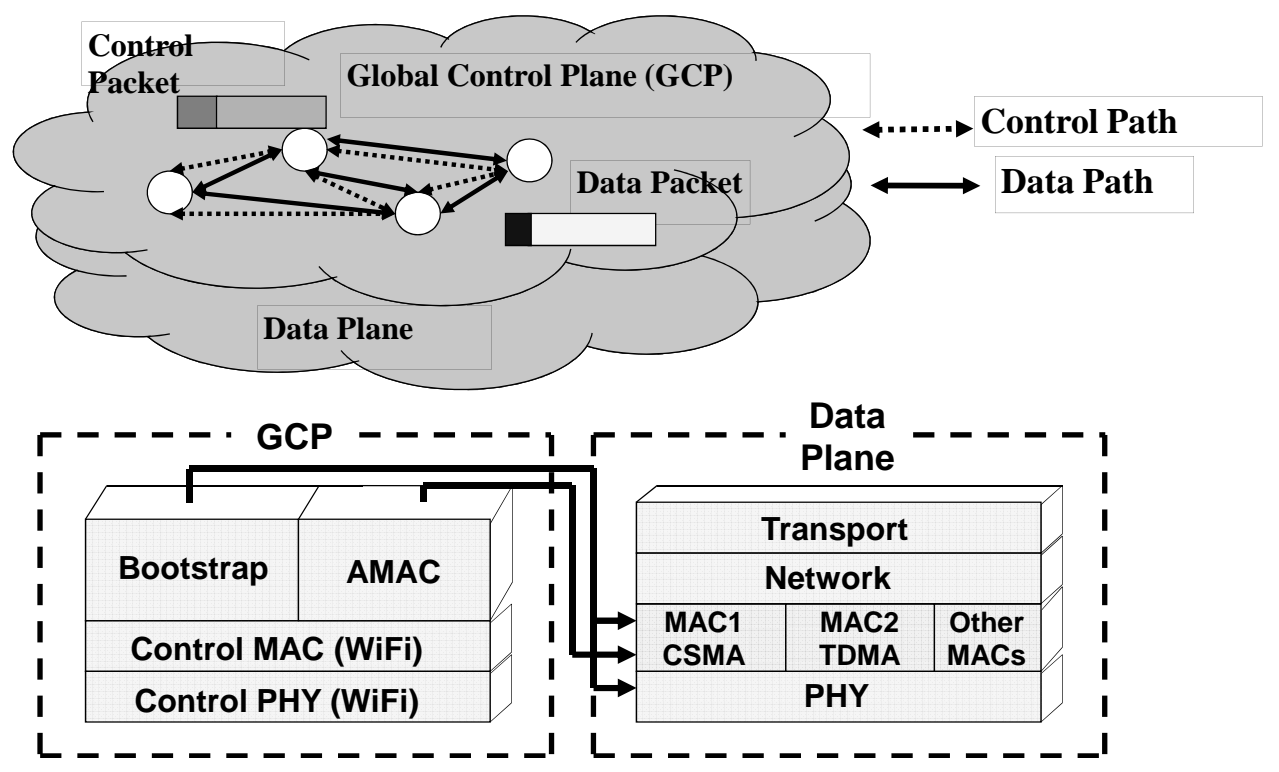

Figure 3.1: GCP and Data Plane structure 


\section{Chapter 4}

\section{AMAC Protocol}

The flexibility offered by cognitive radios makes it possible to design adaptive wireless networks that adjust the protocol stack used based on observed external conditions or service requirements. For example, in a vehicular scenario, there can be large variations in radio node density as cars move from an urban intersection to a highway. CSMA/CA protocol might work well in certain situations when the number of nodes is small, but it may be beneficial to switch to alternative protocols such as TDMA to avoid instability and hidden-node problems when the number of neighbor nodes increases

dramatically. Additionally a separate control protocol can assist with seamless roaming across networks supporting different network conditions. Thus, we develop a protocol framework for dynamic adaptation of MAC layer protocols, considering the examples of switching between CSMA/CA and TDMA.

(1). AMAC architecture

This "Adaptive MAC Protocol (AMAC)" mainly focuses on switching among different MAC protocols to achieve better overall network performance. The proposed AMAC protocol is able to incorporate channel coordination when available vacant channel is detected and to adjust MAC/PHY configurations according to different service requirements and network node density. The GCP outlined earlier provides a control framework to set up network adaptation functions.

It is important for cognitive radio nodes to start with bootstrapping and discovery process in this GCP-based framework. The bootstrapping function can help configure PHY/MAC capabilities and current status when the nodes power up. After initialization, a discovery protocol is executed to provide end-to-end reachability and determine optimum path information across multiple hops. Based on these exchanged control 
messages, nodes are able to initiate data transmission.

The proposed AMAC protocol is used to apply different operating conditions in which wireless nodes can achieve efficient data communication. It dynamically changes MAC behavior on-the-fly and configures per-hop PHY parameters of the data plane at each intermediate node. AMAC includes three phases as following:

- Baseline MAC selection: Each node starts with the MAC protocol which handles the nominal light network traffic condition (CSMA is used in this paper).

- PHY adaption: Nodes first try to discover if they can adapt PHY parameters (such as operating channel, power or modulation type) when the performance of data transmission drops significantly.

- MAC adaptation: When the PHY adaptation is not able to meet performance goals, nodes may initiate a switch of the MAC protocol. AMAC provides a mechanism to let each node negotiate with others and find the suitable MAC protocol commonly used for different network environments.

When the cognitive radio node powers on, it first listens and collects control beacon messages from nearby nodes by using GCP. If the node decides to join the network, it starts by flooding control messages in the GCP to form the initial network connections. Beacon message includes required information of nodes states and ID. In Table 4.1, we show the format of periodic beacon messages (operating MAC, frequency and power) which update neighboring radios with a nodes latest parameters.

\begin{tabular}{|c|c|c|}
\hline Type & Flags & Sender ID \\
\hline \multicolumn{2}{|c|}{ Beacon Transmit Power } & Max PHY Rate \\
\hline \multicolumn{2}{|c|}{ Operating Frequency } & Operating MAC \\
\hline
\end{tabular}

Table 4.1: GCP beacon message format

The key requirement in choosing a baseline operating MAC is to ensure flexible date communication. For instance, CSMA-based MAC is simple, flexible and able to handle most networks with low traffic load; TDMA-based MAC has a fixed structure and avoid 
repeated channel contentions for scenarios with stream traffic. As a result, we choose CSMA as the baseline MAC and consider switching to TDMA when performance is degraded due to excessive contention.

Node mobility in vehicular environments can result in major changes to the propagation environment, radio density and network topology. Cognitive radio nodes are capable of adjusting PHY parameters (e.g. frequency, power, rate, modulation) on the fly when the performance degrades. Each transmitting node will monitor performance (i.e. throughput or delay) of data transmission periodically. When the performance requirement goes below specified objectives, the nodes will initiate PHY parameters adaptation via the GCP to negotiate suitable configurations, such as switching to empty channels or power adjustment.

When PHY adaptation alone fails to meet performance objectives, the next level of adaptation involves change of the MAC protocol. AMAC grants cognitive radio nodes a further way to solve degraded performance caused by contention. When there are nodes initiating video streaming in a high network density area, it may be hard to satisfy the required QoS with PHY adjustments. Instead, if the nodes can adjust the MAC protocol with time or frequency schedule management (i.e. TDMA or FDMA), the desired QoS can potentially be achieved. As another example, a mobile cognitive radio moving from a sparse rural area to an urban can switch between two MAC layer protocols (such as CSMA and TDMA) to deal with such major changes in operating environment.

However, if all the cognitive radio nodes change their MAC protocol based on a local decision, the protocols used by all nodes may not be compatible. Thus, we introduce a voting scheme for nodes to agree on the MAC protocol to be used across the whole network. While a sender requests MAC switching based on previously outlined algorithms, it first broadcasts in the GCP about which MAC protocol it would like to apply. The nodes receiving this "MAC switch request" message will respond with a GCP message indicating their local decisions. In this way, nodes vote for the MAC protocol to be used and the common protocol for the network is determined by the majority nodes. The control message format for the voting scheme is shown in Table 4.2. 


\begin{tabular}{|l|l|l|}
\hline Type & Flags & Sender ID \\
\hline \multicolumn{3}{|c|}{} \\
\hline
\end{tabular}

Table 4.2: Control message format for MAC switching request

Indeed, the above approach can assist with deciding common MAC protocol in smaller network. For the larger network, we believe the voting scheme requires excessive amount of time to collect the local decisions and is not suitable to force quiet amount of nodes to change to other MAC protocols. Therefore, we present a cross-layer design strategy to let end-to-end link use the same MAC protocol without forcing other links to use the same MAC protocol in the same network. The basic concept of this approach is we combine channel assignment with MAC protocol, which means the pre-defined subchannels are only dedicated to some pre-assigned MAC protocols. In the case of CSMA and TDMA MAC, we assign one channel dedicated to TDMA MAC and the others for CSMA MAC. According to the above scheme, if the nodes transmitting data with CSMA MAC request to switch, they will first to switch other CSMA MAC channels. Unless the CSMA MAC channels are not able to satisfy the nodes' requirement, then they will switch to TDMA dedicated channel to maintain the performance. Thus, each node does not require to reach the common MAC and this scheme will not over load the control channel either.

(2). MAC Switching Schemes for $A M A C$

Here, we propose three strategies to help cognitive-radio nodes decide whether to request MAC switching.

- performance degradation threshold

- predicted future traffic patterns

- network density

In the first approach, each node computes its performance in an observation interval while data traffic flows continue. If performance is degraded below some threshold, the node will request a MAC switch. In this approach, we focus on maintaining the node's 
performance in order to satisfy application requirements such as throughput or delay. In the second approach, each node checks its future traffic patterns by periodically examining the MAC transmit buffer. If the average packet size is large, the node may request a TDMA MAC switch in order to reduce contention. On the other hand, for smaller average packet size light load, CSMA is preferable to prevent unnecessary wastage of slot time. Besides, AMAC is able to provide better performance under conditions of different traffic types. Bursty traffic, for instance, can be transmitted efficiently with CSMA; however, streaming traffic is better suited for TDMA transmission because of improved reliability and lower contention.

In the third approach, GCP enables each node to detect the network density and topology in a mobile environment. With AMAC, each mobile node can adapt suitable MAC to different networks or requirements on the fly without degrading the performance of neighbor networks. Using a single MAC everywhere may cause lots of contention or inefficiency in certain mobility scenarios such as vehicles moving from dense city areas to sparse suburban environments.

An example network of data traffic starting from the sender to the receiver with AMAC MAC capability handling both CSMA and TDMA modes is shown in Figure 4.1. During the bootstrap process, all the nodes are able to obtain a global view of the network by exchanging beacons. After data traffic is initiated, the sender node uses CSMA as its baseline MAC protocol. In the mean time, the sender computes the average throughput of its packets periodically (e.g., an average of ten packets). When the performance in the observation interval drops more than $20 \%$ compared to the previous observation interval, the node will initiate a request to switch to TDMA using the GCP. When all its neighbor nodes receive the request, they vote on whether to switch to the proposed MAC protocol based on their local throughput performance. As shown in Figure 4.1, node A, C and Receiver all agree to switch to TDMA and node B votes to keep using CSMA. In the end, the TDMA MAC will be switched on for all the nodes based on the majoritys voting decision. 


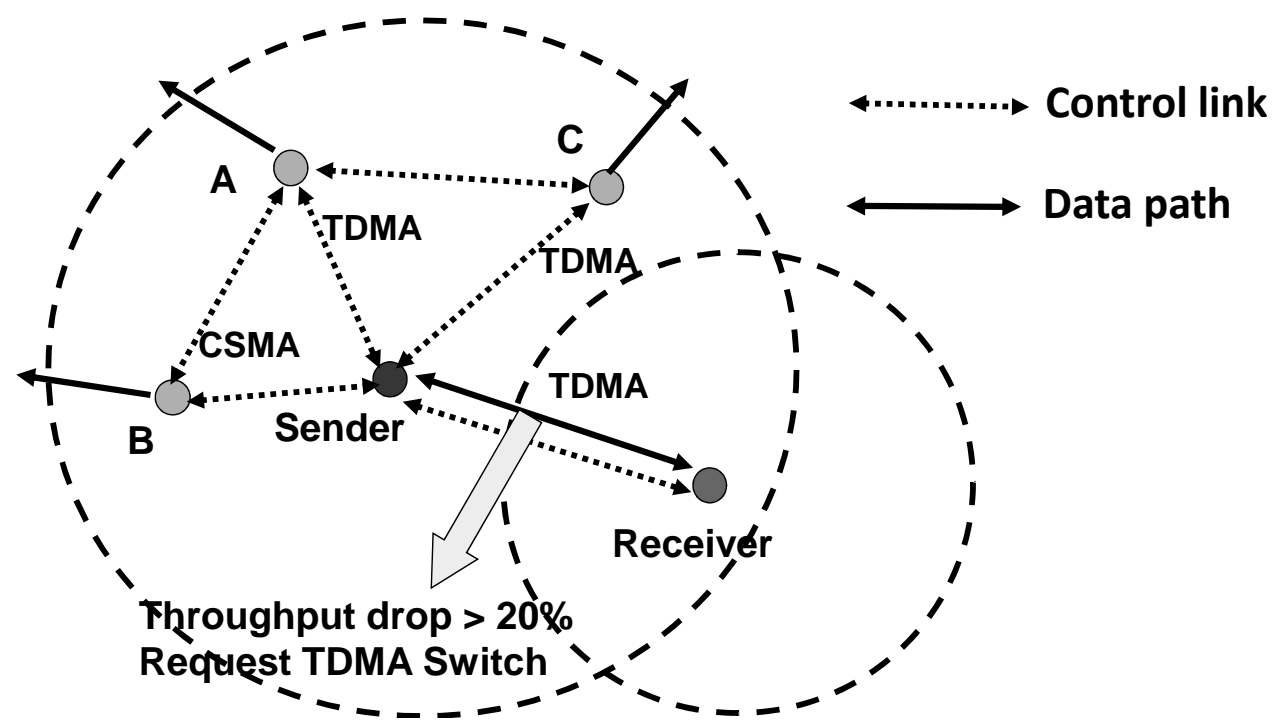

Figure 4.1: Example network of AMAC protocol 


\section{Chapter 5}

\section{SIMULATION RESULTS}

In this chapter, we present the simulation results for the AMAC algorithm using the GCP framework. We use the NS-2 simulator to evaluate the above design for representative usage scenarios. Note that the default NS-2 model is modified to two interfaces control and data interface. The control MAC is set up as 802.11 CSMA and data MAC as AMAC in comparison with CSMA and TDMA. Based on the above configuration and parameters in Table 5.1, CBR/UDP and FTP/TCP traffic are generated at the data interface to evaluate the performance of AMAC. In AMAC, we implemented two candidate MACs - CSMA/CA and dynamic TDMA. The CSMA/CA MAC is similar to the 802.11 MAC but without RTS/CTS reservations. For dynamic TDMA, the first slot of a frame is used for synchronization and to check how many nodes have packets to transmit. Therefore, the slot number of each frame can be adjusted to match the number of transmitting nodes without wasted channel time.

\begin{tabular}{|l|l|}
\hline Topology size & $1000 \mathrm{~m} \times 1000 \mathrm{~m}$ \\
\hline Data Channel rate & $11 \mathrm{Mbps}$ \\
\hline Control Channel rate & $1 \mathrm{Mbps}$ \\
\hline PHY rate & $2 \mathrm{Mbps}$ \\
\hline Carrier Sensing Interval & $20 \mathrm{~ms}$ \\
\hline Propagation Model & TwoRayGround \\
\hline Data Packet Size & 1000 Bytes \\
\hline Path loss index & 4 \\
\hline
\end{tabular}

Table 5.1: GENERAL SIMULATION PARAMETERS

For the performance evaluation, we consider the following scenarios:

1. Mixed Traffic Pattern: Each node is fixed at a randomly-deployed location in a $20 \mathrm{~m}$ by $20 \mathrm{~m}$ area. We generate the CBR/UDP traffic flows between any two 
nodes and each flow generates two different traffic patterns - bursty and streaming traffic.

2. Mobile Environment (type A): In this scenario, a group of mobile nodes move from a low-density suburban area to a high-density urban area. Mixed CBR/UDP and FTP/TCP traffic are generated by the mobile nodes while FTP/TCP traffic is generated by the city and suburban nodes. In order to evaluate the MAC switching strategies, we consider only one available channel without PHY adaptation in the first two scenarios.

3. Mobile Environment (type B): In this more complex mobile service scenario, we implement two available channels in this network and one is dedicated for TDMA MAC. Three groups of mobile nodes drive with each other and a group of static nodes are nearby. VoIP, streaming data and web browsing service are generated by the nodes, and the delay requirement of VoIP has to be satisfied.

\subsection{Scenario with Mixed Traffic Pattern}

Before this experiment, let's first explore the characteristics of CSMA and TDMA MAC to see which MAC protocol is preferable to what environment. We conduct two experiments to evaluate TDMA and CSMA MAC characteristics. One experiment is to generate CBR/UDP streaming traffic with an offered load 2Mbps for each flow and the number of flows is increased. The other experiment is to simulate bursty traffic by providing each flow a 10Mbps CBR/UDP load for 1 second and then 100kbps CBR/UDP load for the rest of time. We increase the number of flows and each flow takes turn to transmit bursty data which means the bursty 10Mbps load won't be overlapped in this network.

According to the Figure 5.1, for lighter traffic loads, TDMA and CSMA have similar throughput. However, the more the flows generated, the higher throughput TDMA MAC has compared to CSMA MAC (yields more than twice throughput with 100 flows in the network). That is because the increase of the flows causes contention increase and then drops CSMA MAC performance. For bursty traffic scenario, we generate 


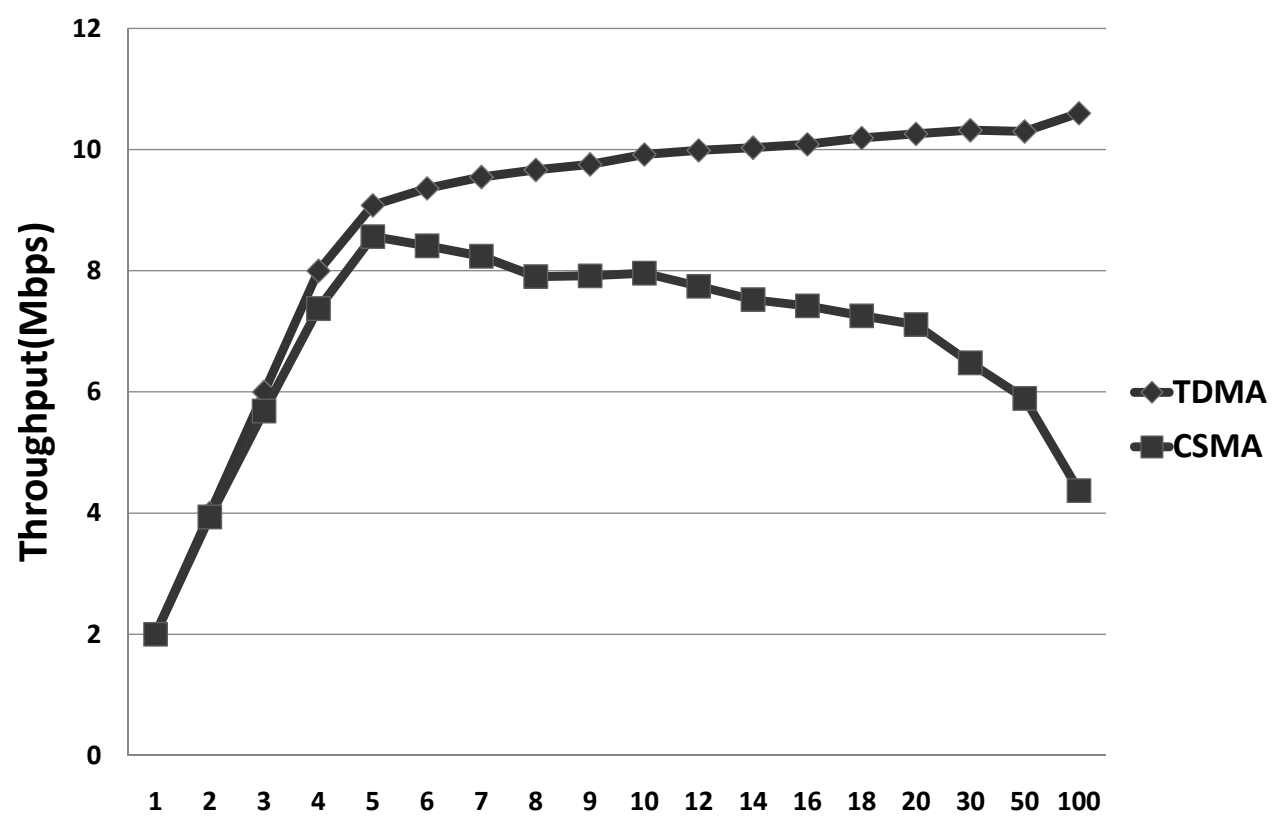

Figure 5.1: Characteristics of CSMA and TDMA with streaming traffic

10Mbps CBR load for one second and the rest is 100kbps CBR/UDP load for each flow. In the network point of view, each flow takes turn to transmit this one second 10Mbps load which means there won't be overlapped 10Mbps load in any second of data transmission. In Figure 5.2, the CSMA MAC outperforms TDMA MAC since the bursty traffic flow with CSMA MAC is able to keep transmitting packets out while the other flows are in low traffic. With TDMA MAC, each flow has to wait till its own slot time and then transmit packet so it is unable to handle bursty traffic in time and instead drop them. Based on the above result, this simulation runs with 40 nodes and generates each flow as mixed CBR/UDP traffic (bursty and streaming traffic) which is shown in Figure 5.3.

In Figure 5.4, we evaluate 8, 10 and 12 flows in this network. It can be noticed from previous results that the more flows in streaming traffic, the bigger throughput difference between TDMA and CSMA. However, for bursty traffic scenario, if the flow number keeps increasing, the packet collision will be increasing, which cause CSMA performance drops and the throughput difference is getting less. In order to reach better AMAC performance gain, we choose 8 to 12 flows heavy traffic load in this scenario. Since each flow combines different traffic types, the total average network 


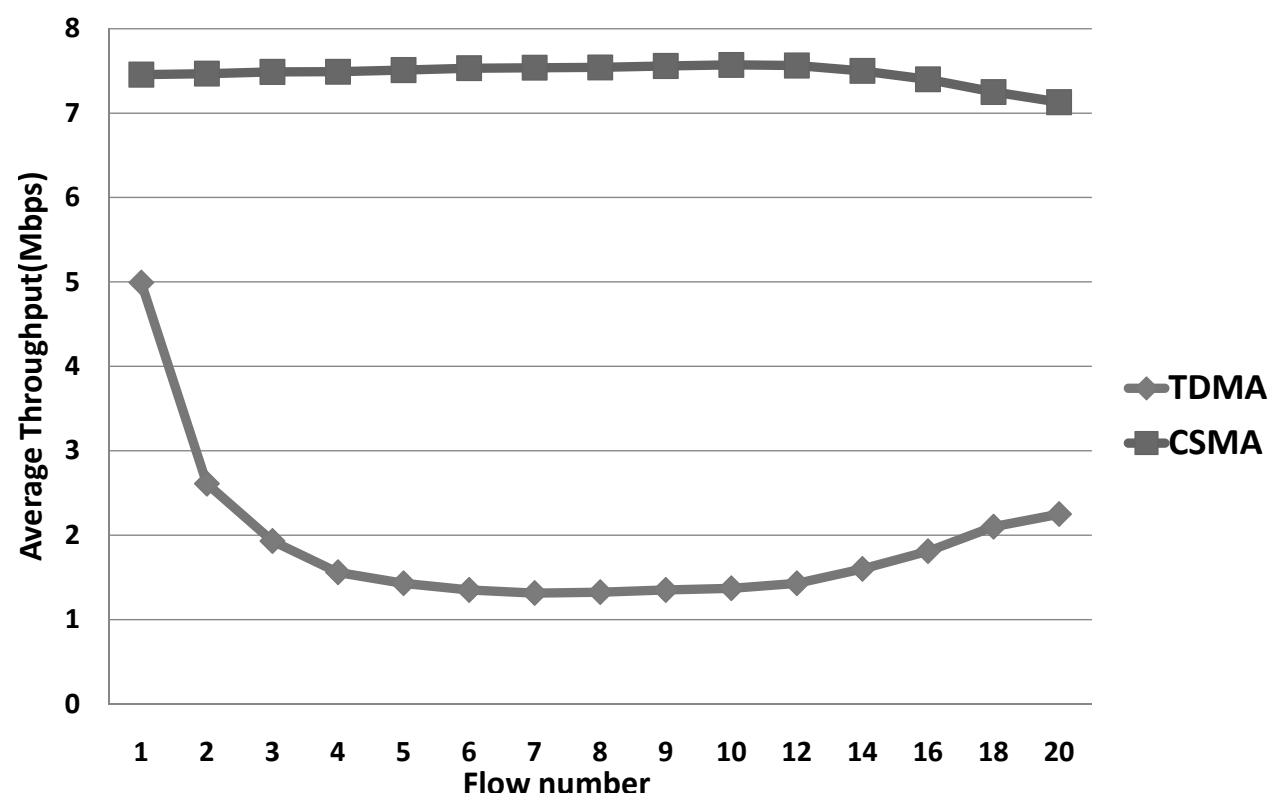

Figure 5.2: Characteristics of CSMA and TDMA with bursty traffic

load is controlled to be around $20 \mathrm{Mbps}$ in each case so as to avoid overloading the channel. It can be noted that the average throughput of CSMA is a little better than TDMA because CSMA is able to transmit more packets for bursty traffic even though TDMA performs better in streaming traffic. The AMAC algorithm detects the "packets incoming rate" in the MAC buffer as switching threshold. If the rate is high, the node will request TDMA as baseline MAC; In other cases, CSMA will be selected. During bursty traffic transmission, because of non-overlapped bursty load in the network, CSMA will tend to be selected by majority vote. TDMA will be chosen as the common MAC while many nodes initiate $2 \mathrm{Mbps}$ streaming load. The result shows the average throughput improves 20\% compared to static TDMA and 15\% compared to static CSMA. This is because AMAC adapts to choose the best MAC for different traffic types.

\subsection{Scenario with Mobile Environment (type A)}

In a vehicular scenario, there can be large variations in radio node density as mobile nodes move from an urban intersection to a suburban highway. CSMA/CA might work well in certain situations when the number of nodes is small, but it may be beneficial 


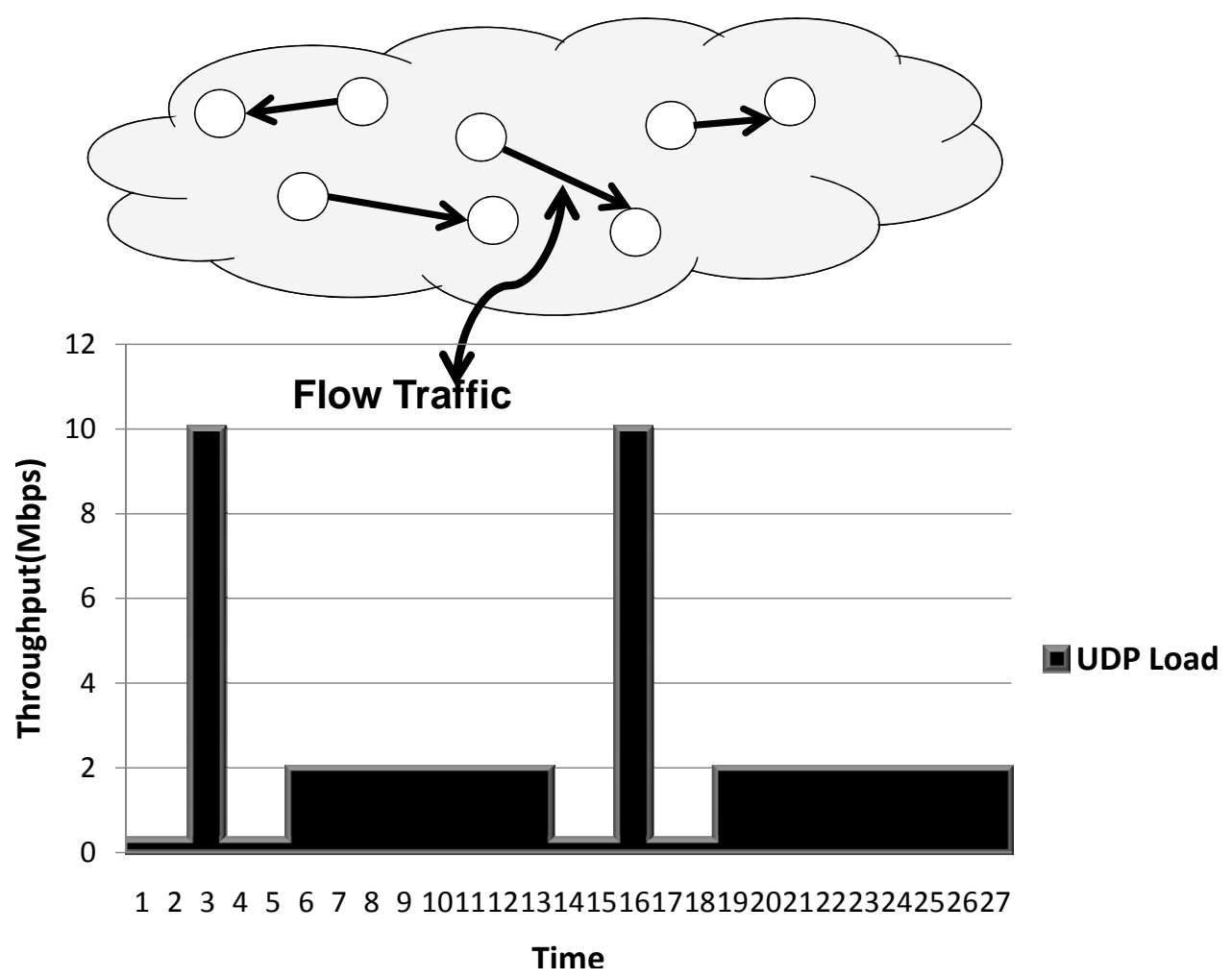

Figure 5.3: Flow traffic type in mix traffic scenario

to switch to alternative protocols such as TDMA to avoid instability and hidden-node problems when the number of neighbor nodes increases dramatically. To study this, we generate a scenario in which 6 mobiles drive from suburban area (CSMA-based network) to city area (TDMA-based network) at 60 miles/hr speed, which is shown in Figure 5.5. For mobile nodes, FTP/TCP and bursty CBR/UDP traffic are generated by the nodes. In the city and suburban area, we create 12 and 1 FTP/TCP flows respectively to represent the difference of node density.

Considering three situations for the mobile nodes. First, if CSMA is used in mobile network, the mobile nodes won't affect other CSMA-based network but they cannot send any packets out when moving to a TDMA-based network region because of no free medium for newly arriving mobile users. Second, if TDMA is used in the mobile network, the mobile nodes are not able to utilize the medium well in CSMA-based network especially for bursty CBR/UDP traffic. However, when these mobile nodes move to a TDMA-based network region, they can join the network and acquire time slots to transmit packets. The city network nodes may sacrifice a little throughput 


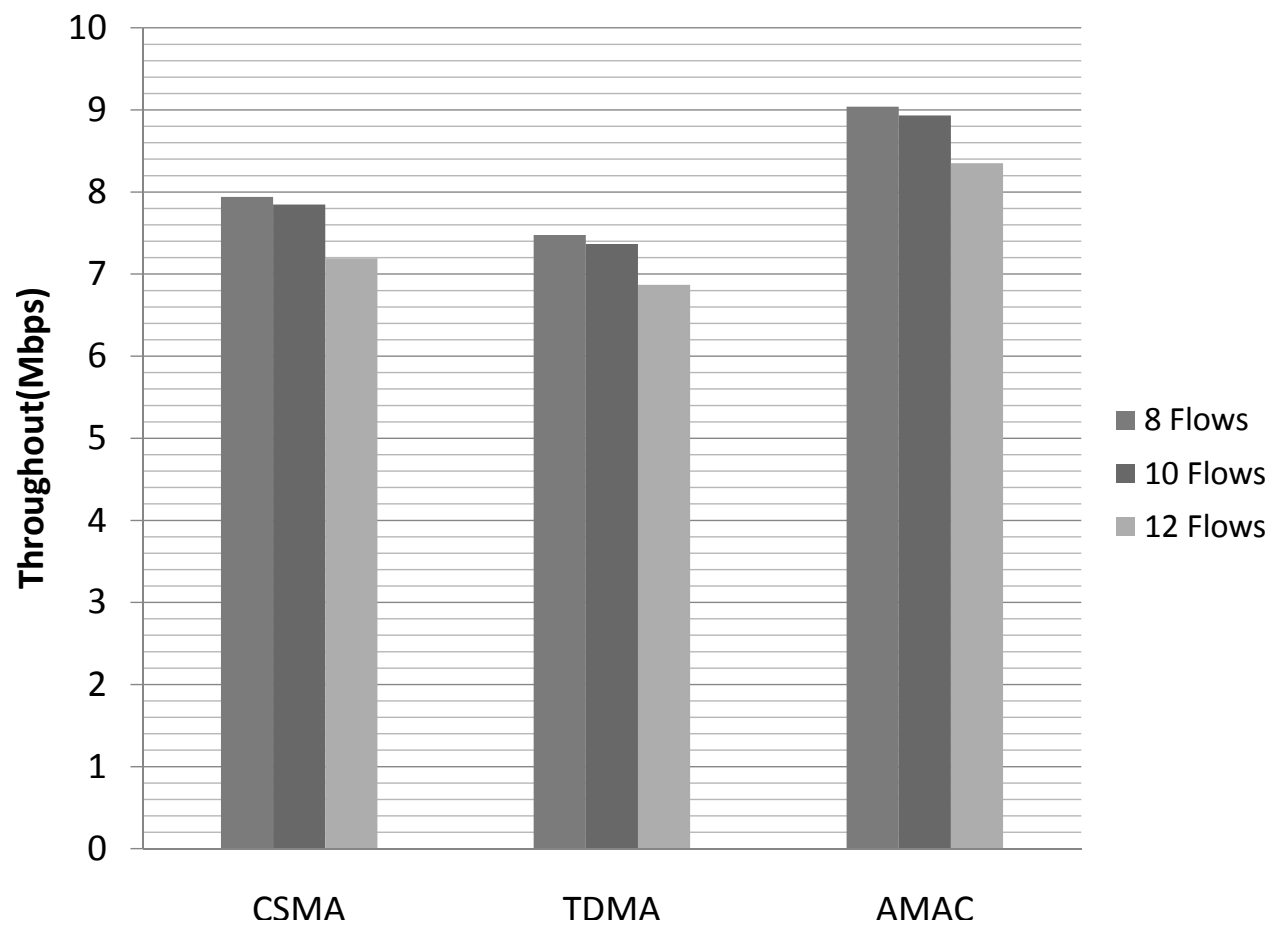

Figure 5.4: Average throughput of mix traffic scenario

by reassigning time slots to mobile nodes but both mobile and city nodes are able to send data out without collision. The final case is that of using AMAC offers the advantage of switching between CSMA in low-density area and TDMA in high-density places. The bursty traffic can fully utilize the medium by CSMA which is also able to transmit packets with TDMA when moving to the city area. The mobile nodes with FTP/TCP transmission can also utilize the medium efficiently by adapting to suitable MAC protocols. It can be seen that when mobile nodes get close to the city area, they detect another MAC network existing by GCP beacons. The request to join the TDMA-based network will be distributed in the control channel and the new scheduled time slots will start by the next frame. The result is shown in Figure 5.6. We observe that AMAC improves the average throughput of this mobile network by $20 \%$ when compared to static CSMA and TDMA and the performance of city and urban networks are maintained at the same time. 


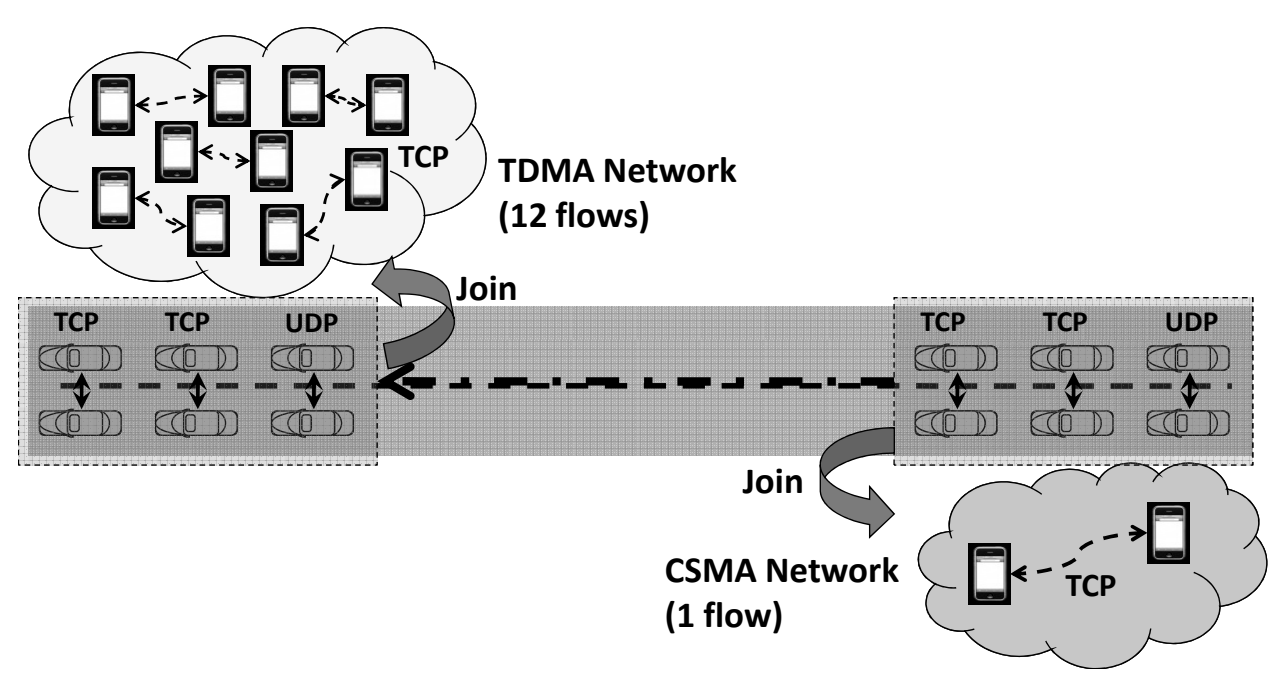

Figure 5.5: Mobile scenario with different MAC-type networks

\subsection{Scenario with Mobile Environment (type B)}

In the Figure 5.7 scenario, there are three groups of mobile nodes driving with each other at 40 miles/hr speed and one phone network close by. VoIP service is applied to one mobile network and the streaming data is for the other two mobile networks. The phone network mainly uses TDMA for web browsing on Channel 2(CH2). On Channel 1(CH1), the VoIP mobile network initiates voice data transmission first and the other mobile networks take turns to join. VoIP data uses 96Kbps UDP streams of 300-byte frames and streaming data has 2Mbps CBR/UDP load with 1000-byte frames. Our goal is to satisfy $30 \mathrm{~ms}$ delay requirement for VoIP data transmission. We consider the following situations for VoIP mobile nodes:

Static CSMA: Based on the previous algorithm, if the $30 \mathrm{~ms}$ delay requirement cannot be achieved, the nodes will request to switch channel to $\mathrm{CH} 2$ (PHY adaptation). However, the fully-loaded TDMA network on $\mathrm{CH} 2$ prevent VoIP nodes transmitting any packets by CSMA and the voice quality drops.

Static TDMA: TDMA ensures the VoIP data delay requirement by assigning the dedicated collision-free time slots on $\mathrm{CH} 1$. But, it scarifies the other two streaming CSMA network performance by stopping their data transmission while TDMA packets are scheduled. 


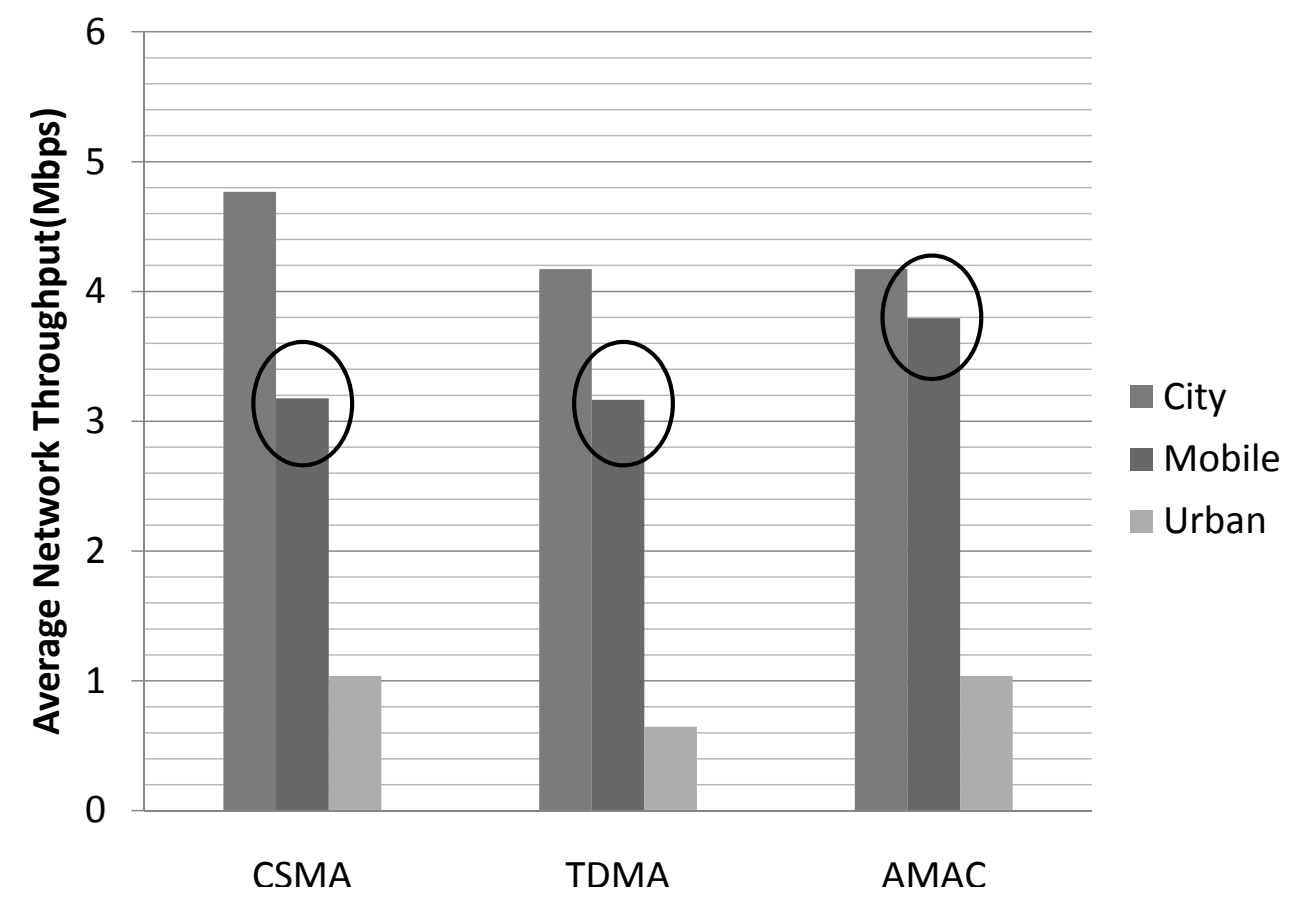

Figure 5.6: Average network throughput of city, mobile and suburban networks

AMAC: While AMAC is applied for VoIP data transmission, it first uses CSMA on CH1. After VoIP mobile nodes detect average delay is more than $30 \mathrm{~ms}$, switching to TDMA in $\mathrm{CH} 2$ will be requested. The time slots in phone network will be reassigned and the delay requirement is able to be satisfied.

In Figure 5.8, we can see that VoIP data transmission using AMAC reaches four times throughput of static CSMA and the 2Mbps streaming data transmission can achieve twice the throughput of TDMA. Although the throughput of TCP flows may have dropped a little, it may be acceptable to trade off best effort web or content applications against real-time voice or streaming video. 
TDMA Network at $\mathrm{CH} 2$

(10 Web Browsing Flows)

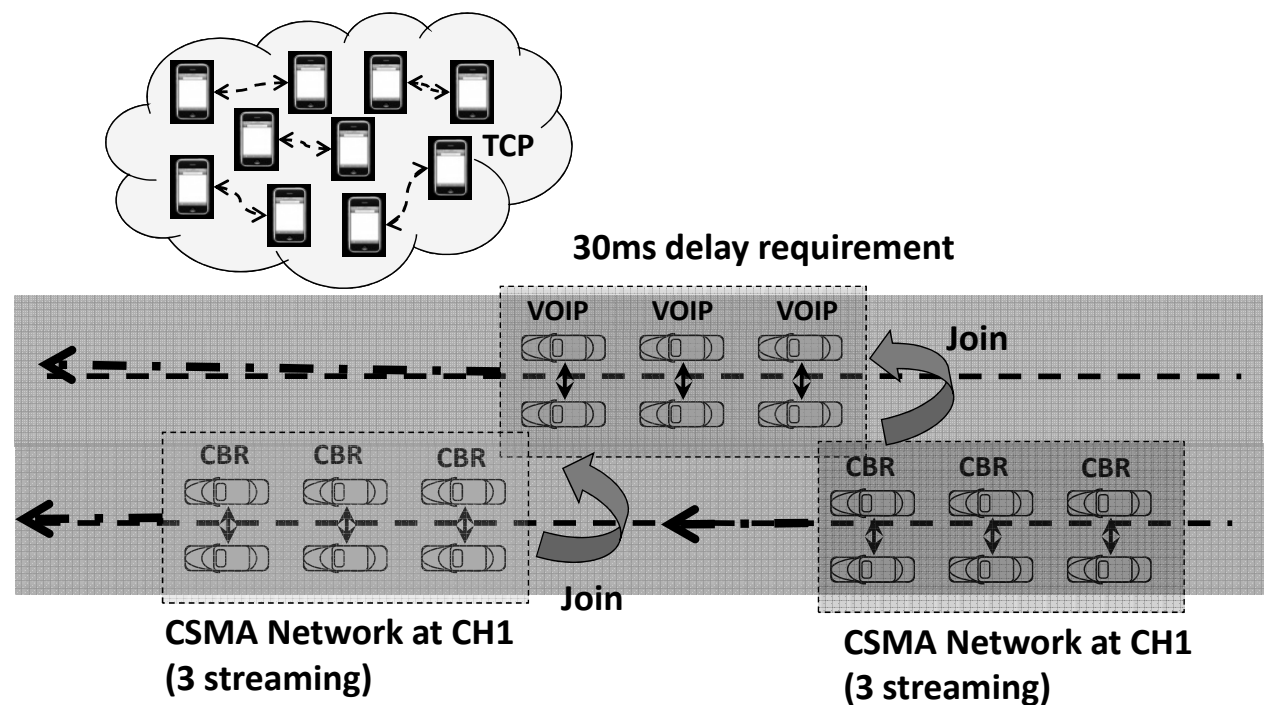

Figure 5.7: Mobile scenario with mix service types

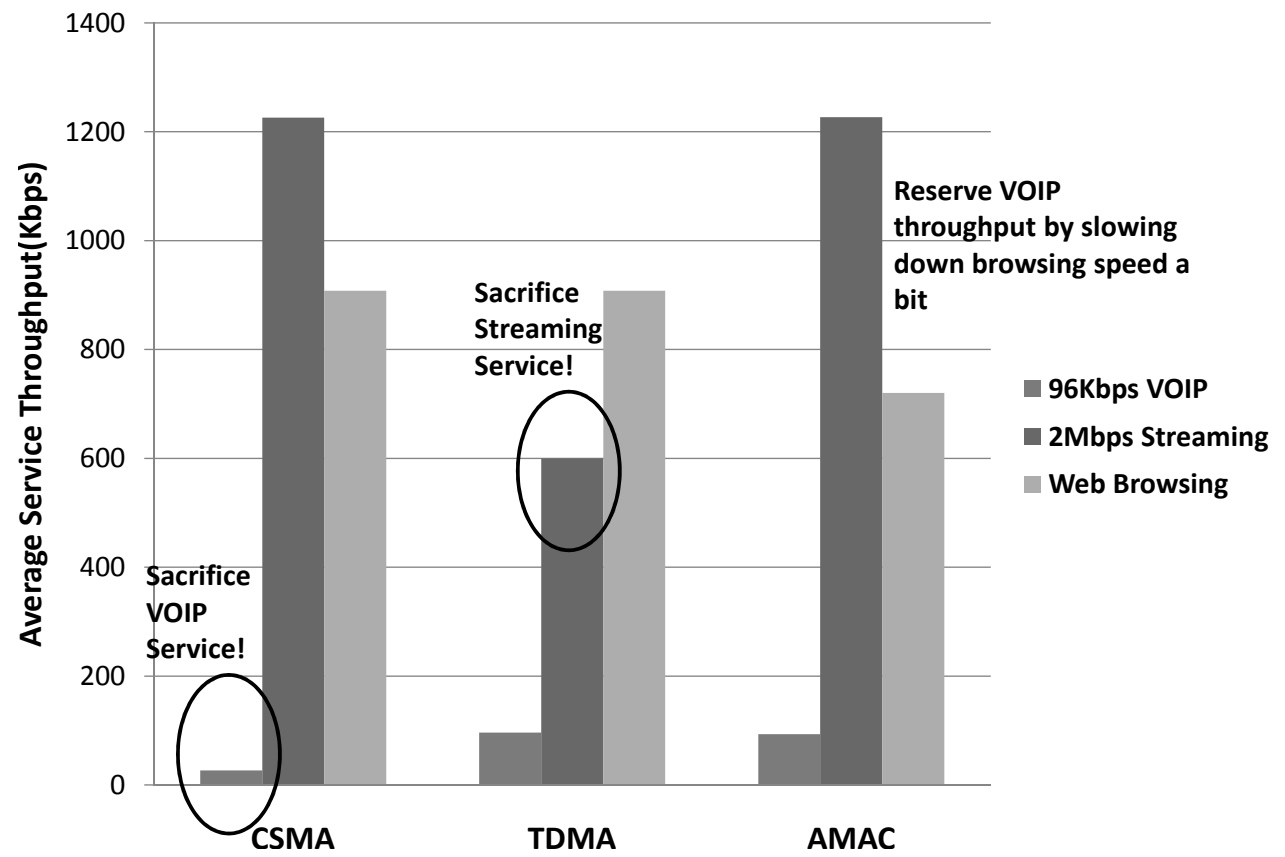

Figure 5.8: Average network throughput of VoIP, streaming and web browsing networks 


\section{Chapter 6}

\section{EXPERIMENTAL EVALUATION ON GNU RADIO/ORBIT}

In this chapter, we present results from experimental prototyping of the proposed AMAC protocol using the GNU/USRP software radio platform available on the ORBIT testbed. In the current experiment, we use five nodes with dual radios (GNUradio $+\mathrm{WiFi}$ ) for proof-of-concept validation. Additionally, to focus on the MAC switching evaluation, we only demonstrate results with AMAC without PHY adaption.

\subsection{Experiment Setup}

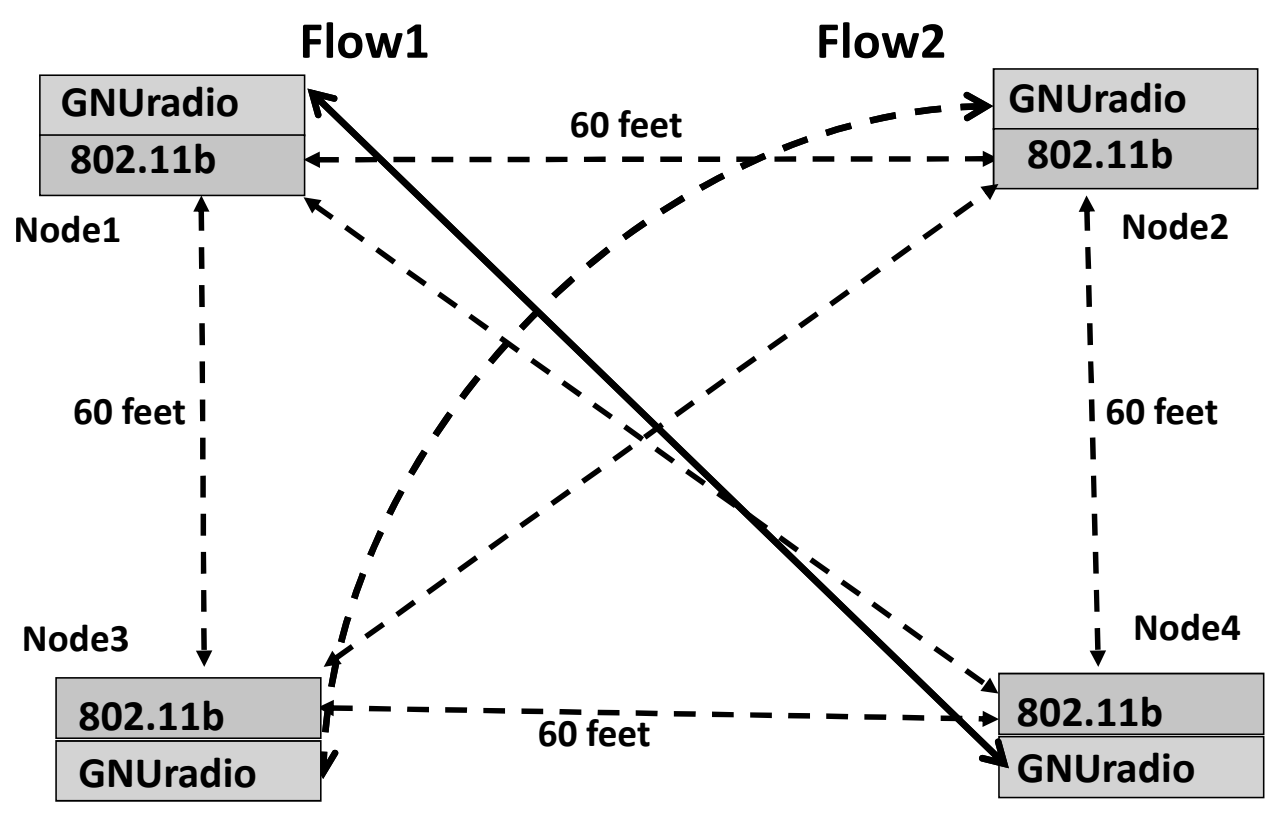

Figure 6.1: Experimental network topology for AMAC

We conducted experiments on AMAC with CSMA to TDMA switching on the GNU radio nodes in ORBIT testbed. The GCP and data plane are implemented with a dualradio structure by using a separate control and data radio in each node. The GCP is 
implemented by $802.11 \mathrm{~b}$ radios operating at $2.4 \mathrm{GHz}$ (which are available in addition to the GNU radio on every ORBIT testbed node) and the data plane is a GNUradio operating at 400MHz. Radio parameters for GNUradio are specified in Table 6.1. Due to the limited processor ability of nodes in ORBIT, we choose 50kbps PHY bit rate as proof-of-concept. Figure 6.1 depicts the $802.11 \mathrm{~b}$ - GNUradio node structure and the network topology. The network scenario includes four dual-radio wireless nodes because of limited number of GNUradio nodes in ORBIT testbed. Each node has same inter-node distances (an average of 60 feet) and same radio configurations. In order to represent the worst-case interference scenario, we let node pair $(1,4)$ as Flow 1 and $(2$, 3) as Flow 2 to represent two different data transmission flows. In the third experiment, we use five-node topology with one 3-node flow and one 2-node flow to evaluate AMAC in the multi-hop scenario.

\begin{tabular}{|l|l|}
\hline & DATA PLANE (GNURADIO) \\
\hline PHY Type & GMSK \\
\hline Operating Freq & $400 \mathrm{MHz}$ \\
\hline PHY rate & $50 \mathrm{kbps}$ \\
\hline MAC Type & CSMA/TDMA \\
\hline \multirow{3}{*}{ Transport Protocol } & $(1)$. UDP with CBR load 25, 50 and 75kbps and \\
& with packet size 500B and 1500B \\
& $(2)$. TCP file transmission \\
& (3). UDP with CBR in Multi-hop scenario with \\
& dynamic application \\
\hline
\end{tabular}

Table 6.1: GNURADIO(Data Plane) radio parameters

In the case of AMAC CSMA/TDMA mode, we implement basic CSMA and coarsegrained TDMA protocol because of limitations to GNU radio timing control. In the CSMA protocol, when the sending node senses carrier, it delays 1ms as baseline and then implements exponential back-off while continuing to sense the carrier. In the TDMA protocol, we let each node synchronize to a central node and design the time frame based on the packets round-trip time. For AMAC, all the nodes first use CSMA protocol as baseline MAC and then request the suitable MAC protocol for the network based on their local decisions after data transmission initiated. Using the voting procedure, the 
node requesting MAC change will collect all the votes and announce the final decision.

\subsection{Experimental Results}

\section{(1) Baseline evaluation with static traffic}

The AMAC algorithm will be evaluated using different traffic types (i.e. UDP and TCP) and switching thresholds. First, since the physical bit rate of the GNU radio is $50 \mathrm{kbps}$, we offer three different UDP traffic loads (25kbps, 50kbps and $75 \mathrm{kbps}$ ) to illustrate CSMA, TDMA and AMAC performance. For TDMA, we choose two possible time slots for two senders and each slot is $300 \mathrm{~ms}$ and $100 \mathrm{~ms}$ for $1500 \mathrm{~B}$ packet size and 500B packet size respectively. For this experiment, we use throughput as the switching threshold. If the performance drops more than $20 \%$ in observation five packet intervals, the sending node will request MAC switching. In our experiment, we let two flows start at the same time to examine the performance of AMAC in the worst-case contention scenario.

Average AMAC throughput measurements for the given 4-node static topology are shown in Figure 6.2 and Figure 6.3. We compare the average throughput of UDP transmission for both small packet size (500B) and large packet size (1500B). The average throughput is computed from the number of packets successfully transmitted over a window of 100 packets. Three different UDP traffic loads, 25, 50 and $75 \mathrm{kbps}$ are shown for comparison. The results show that both TDMA and CSMA have similar performance. For node pair 1, CSMA gives slightly higher throughput and TDMA has better performance for node pair 2 due to different radio activities of GNUradio nodes. Use of AMAC appears to bring up the performance to the higher value between both node pairs. Even though the performance differences are small, the experiment confirms the fact that adaptive MAC switching can provide the upper bound of performance across both MAC protocols. In Figure 6.2 and Figure 6.3, it is observed that AMAC's performance is getting closer and even better than the best performance MAC when the load is increased. Besides, we observe that in real platform, throughput may not be a good MAC switching threshold if not consider about PHY adaptation since some 
of the unpredictable factors, such as radio activity, might have a big influence on the data transmission.

\section{UDP with pkt=1500B}

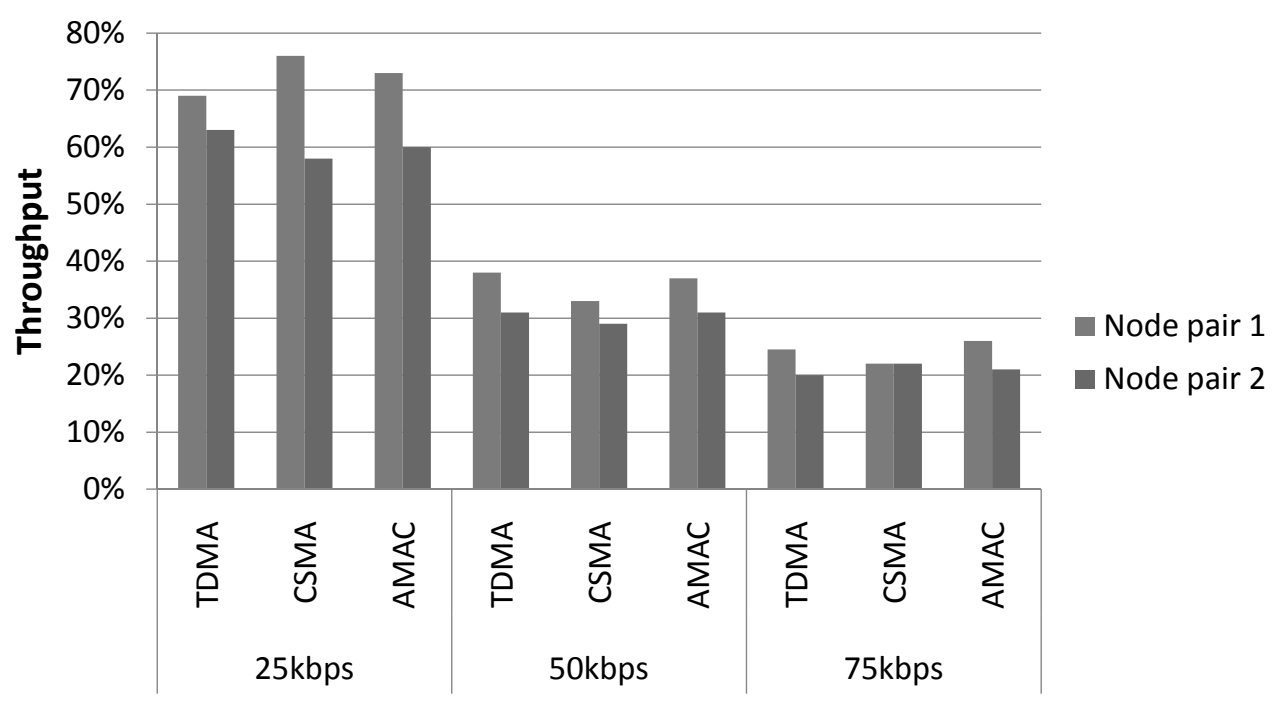

Figure 6.2: Average throughput of UDP traffic with larger packet size

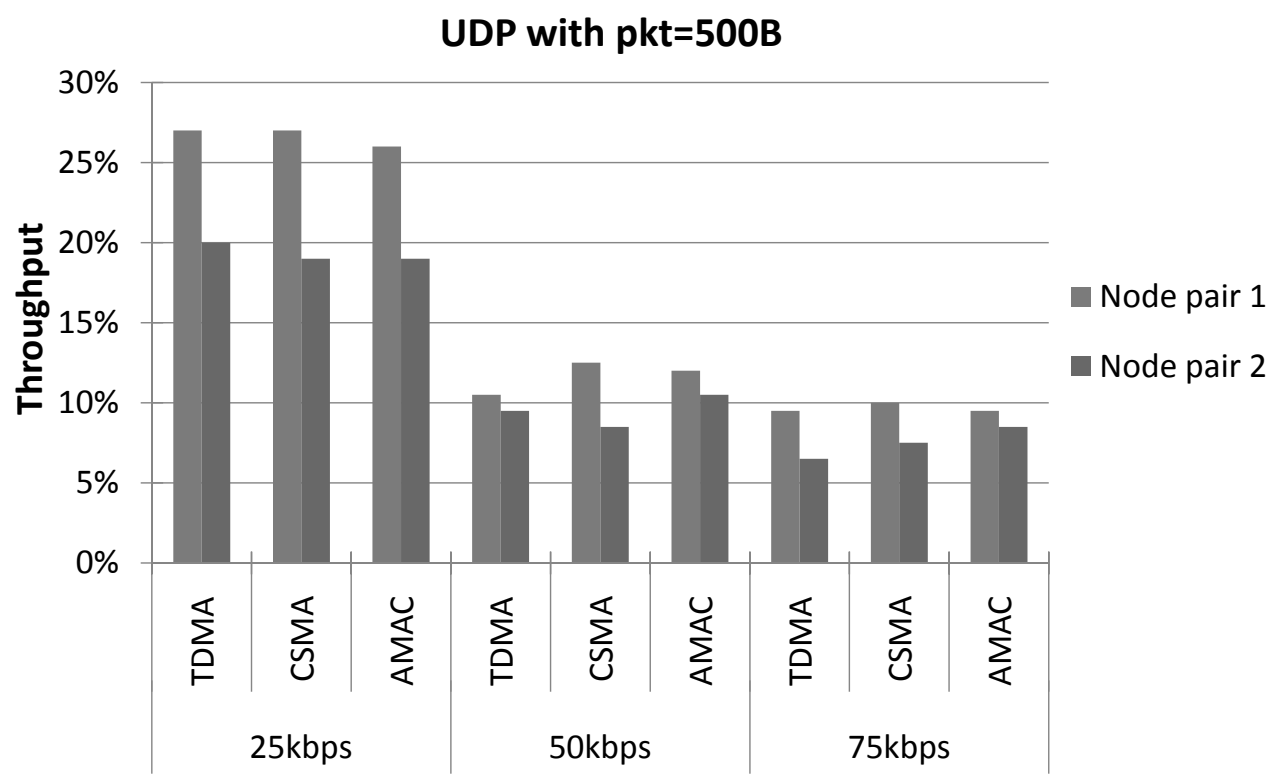

Figure 6.3: Average throughput of UDP traffic with smaller packet size

In Figure 6.4, we show how AMAC responds to changes in UDP traffic with 25kbps CBR traffic load. The results for network throughput vs. time (proportional to number of transmitted packets) are compared for CSMA, TDMA and AMAC. When both pairs of nodes initiate data transmission, CSMA starts to perform worse and the average 
throughput goes down to about $1015 \mathrm{kbps}$. On the other hand, TDMA can always achieve 17-22 kbps because there is no contention between the node pairs. In the AMAC experiment, the network switches total four times over the observation interval. At first, nodes discover throughput drops due to contention so they switch to TDMA. After the 65th packet, in Figure 6.4, TDMA performance drops because of poor radio activity, it then switches to CSMA until the 95th sent packet. Around the 125th sent packet, the nodes switch to CSMA and to TDMA around the 140th sent packet. Since performance drops may be caused by other reasons such as radio activity, AMAC with only MAC adaption may not be able to get better performance. However, AMAC is still capable to track the better performance MAC by adapting between different MAC protocols such as CSMA and TDMA.

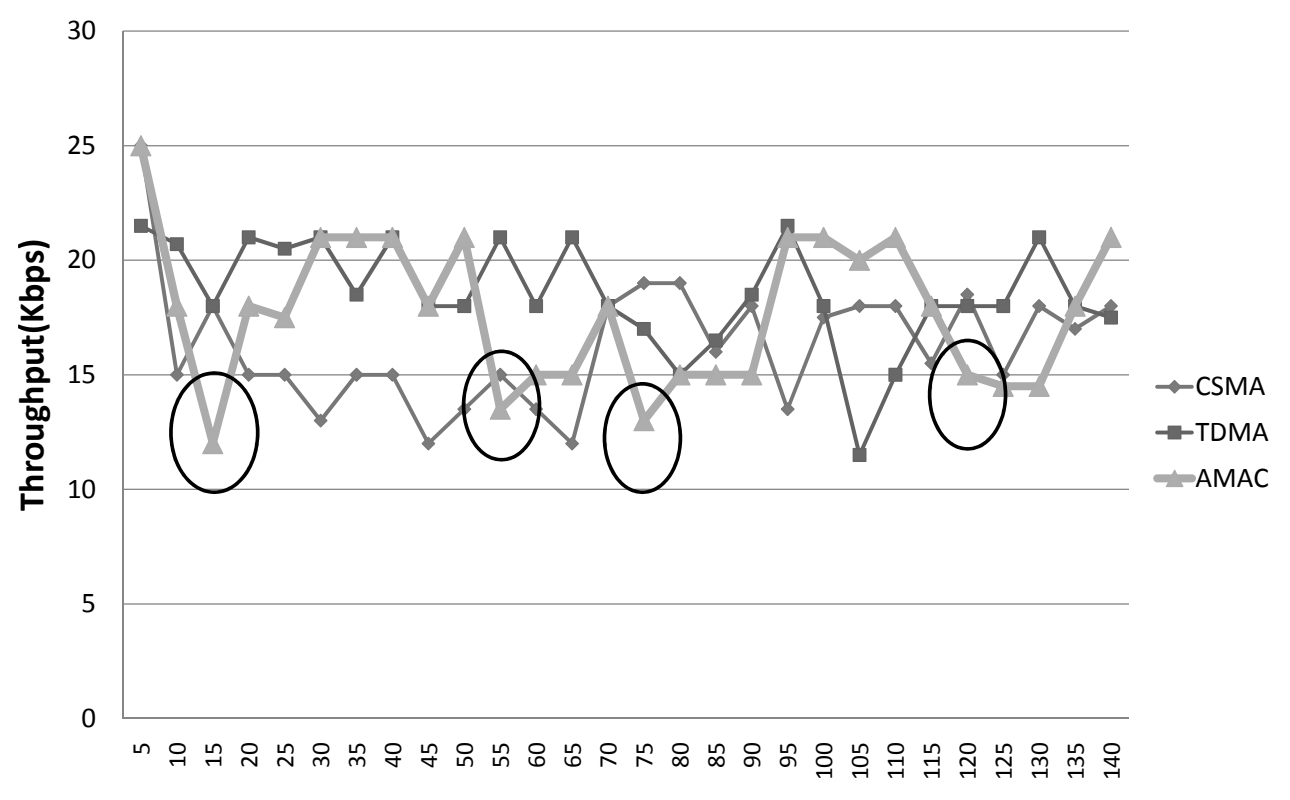

Transmit packet number

Figure 6.4: Average throughput of UDP traffic with smaller packet size

\section{(2) Experiments with realistic dynamic traffic}

In order to quantify the effect of AMAC on practical applications better, we present a file exchange scenario over TCP protocol. We use 4-node topology same as the first experiment. The TDMA time slot for these four nodes is set up as $300 \mathrm{~ms}$ because of GNUradio processing latency ( $50 \mathrm{~ms}$ ) and packet round trip time ( $250 \mathrm{~ms}$ ). Each node still starts with CSMA protocol and detects its application throughput during 
10 seconds observation interval. If it discovers the throughput drops $20 \%$ compared to the throughput of the last interval, a MAC switching request will be initiated and exchanged on the GCP between all the senders and receivers.

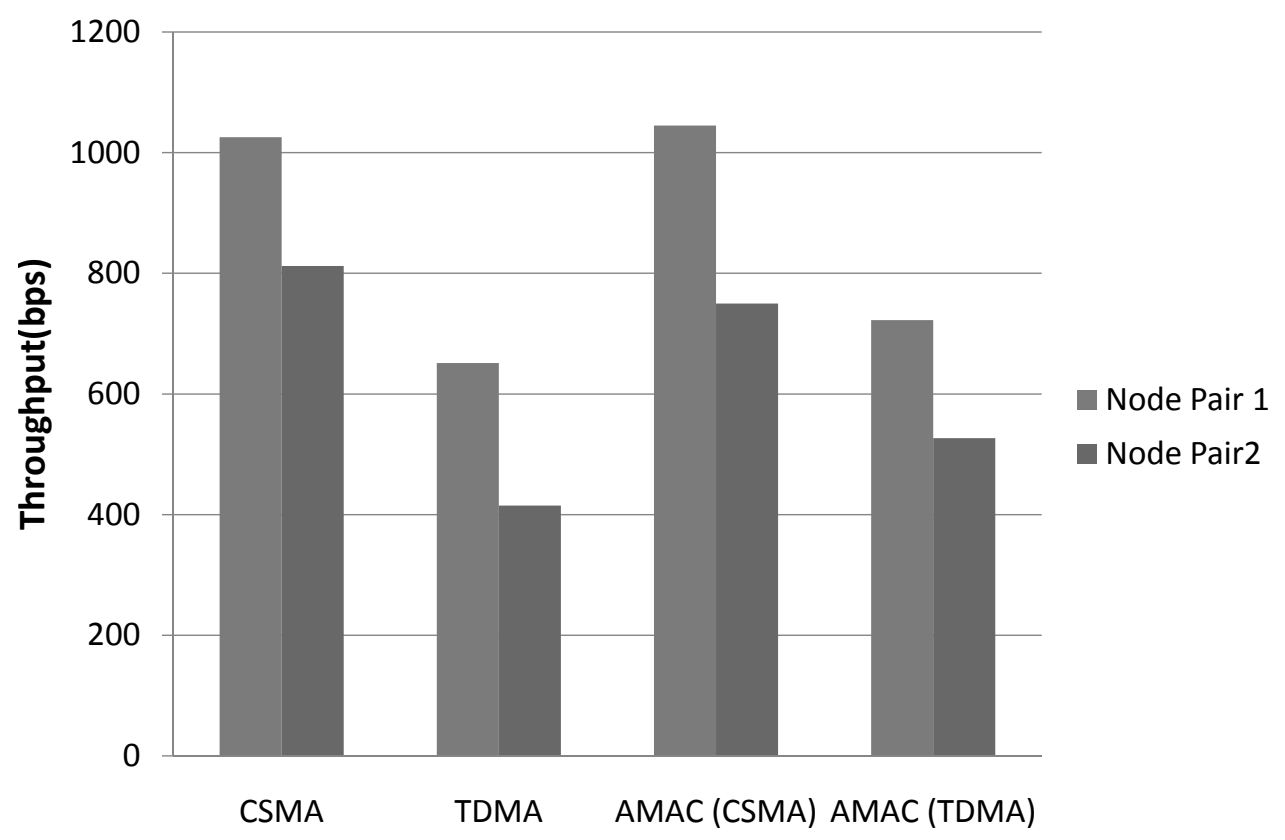

Figure 6.5: Average throughput of CSMA, TDMA and AMAC over TCP transmission

In Figure 6.5, we show the results of using TCP to transmit a 1MB file between these two pairs of nodes. Since TDMA has to have a large time slot and only two pairs of nodes initiate data transmission, the performance of TDMA is worse than CSMA due to the inefficient use of a whole slot taken by TCP ACK packets and larger time slot. Based on the voting strategy, we define AMAC (CSMA) as CSMA favorable mode which means the whole network switches to CSMA as long as at least two nodes decide to use CSMA as their protocol. Similarly, AMAC (TDMA) means the network will apply TDMA when at least two nodes choosing TDMA protocol. With different MACs, we compare the average throughput in Figure 6.5. It is obvious that AMAC algorithm reduces collision by switching to different MAC so no matter which MAC protocol AMAC favors, it is still capable of finding the better MAC to apply at different time. Although the environment is static, the radio activity, packet collision and host processing speed cause unstable performance and then initiate AMAC to adjust to different MAC. AMAC is trying to reach suitable MAC under the network interest. 
(3) Experiments with dynamic applications

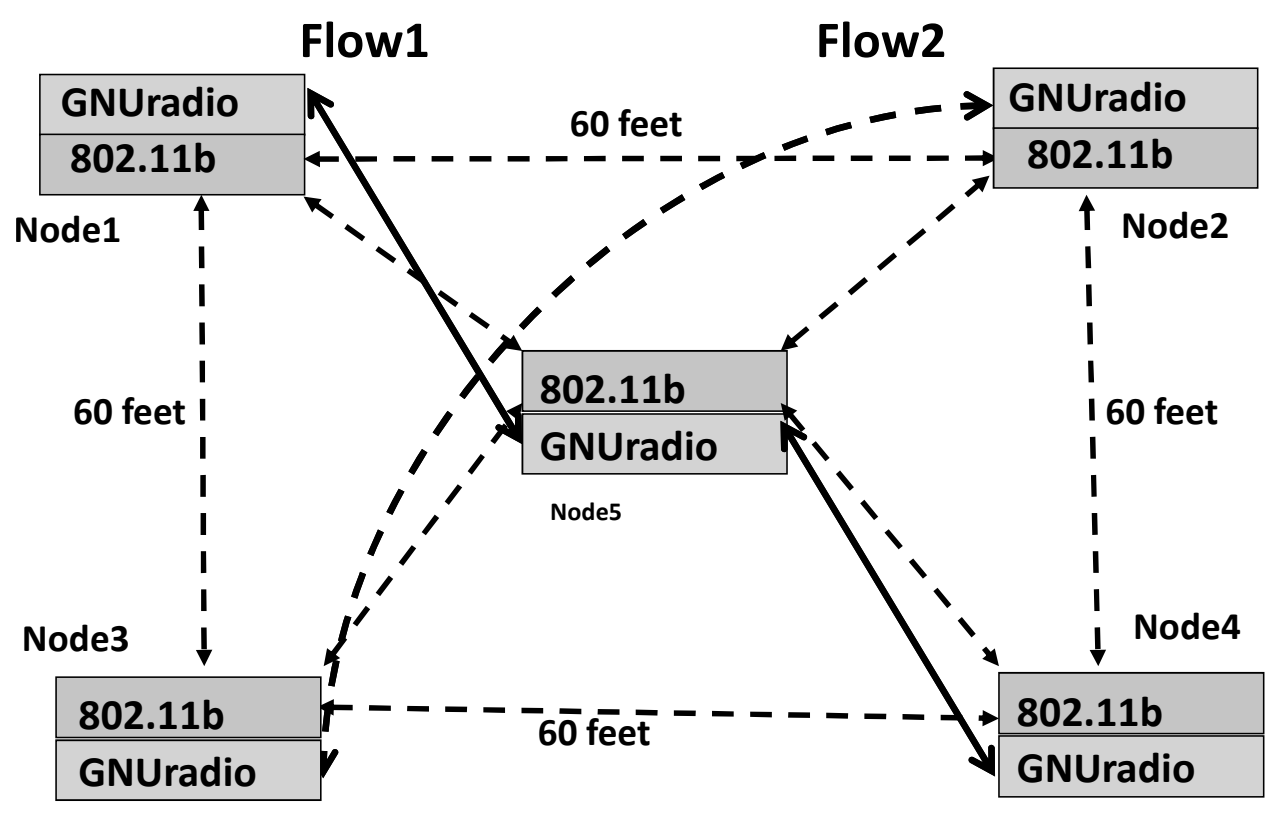

Figure 6.6: Topology of multi-hop scenario

Using performance as AMAC threshold is only one aspect of this algorithm. In this experiment, we conduct a 3 nodes multi-hop scenario for one flow and the other flow is same as before, which is shown in Figure 6.6. AMAC is evaluated with dynamic applications using a mix of short messages and streaming service. The CBR/UDP traffic is generated in two sending nodes. Each sending node starts with CSMA as baseline MAC and switches to TDMA if it detects the average future packets buffer size is more than some threshold such as $800 \mathrm{~B}$, which is the approximate value determined by the half of sum of different packet sizes. Besides, TDMA and CSMA have a similar performance at this value. In Figure 6.7, we present the results of throughput varying with time while long packets (1500B) are followed by short packets (100B). It is to be noted that CSMA has higher throughput during short packet transmission and TDMA has higher and stable performance during long packet transmission. AMAC adapts CSMA for short data at first and when the long streaming data initiates, the sending nodes starts to request MAC switch if the average packet size is larger than $800 \mathrm{~B}$. There is a small time period to let the nodes in the network determine the common MAC. After that, AMAC adapts TDMA for long packets. In Figure 6.8, we can see that the average throughput of AMAC can be achieved $18 \%$ and $20 \%$ higher compared 
to TDMA and CSMA relatively.

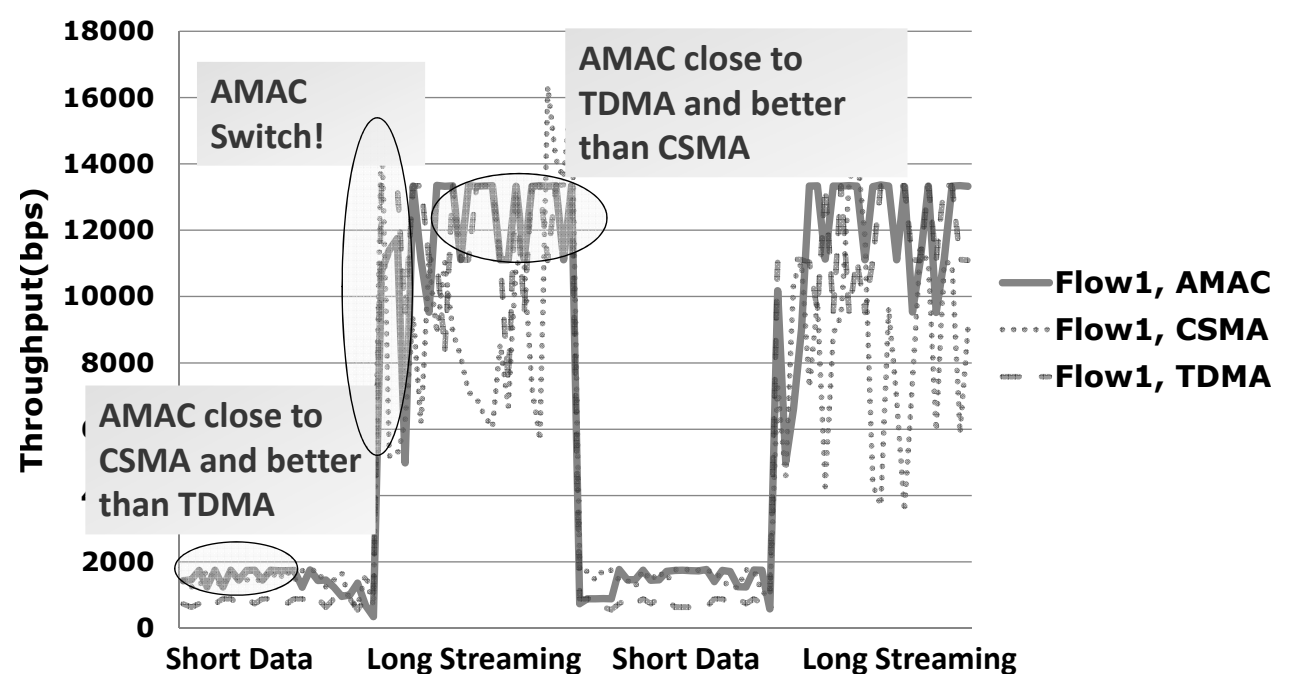

Figure 6.7: Throughput comparison varied with time between CSMA, TDMA and AMAC

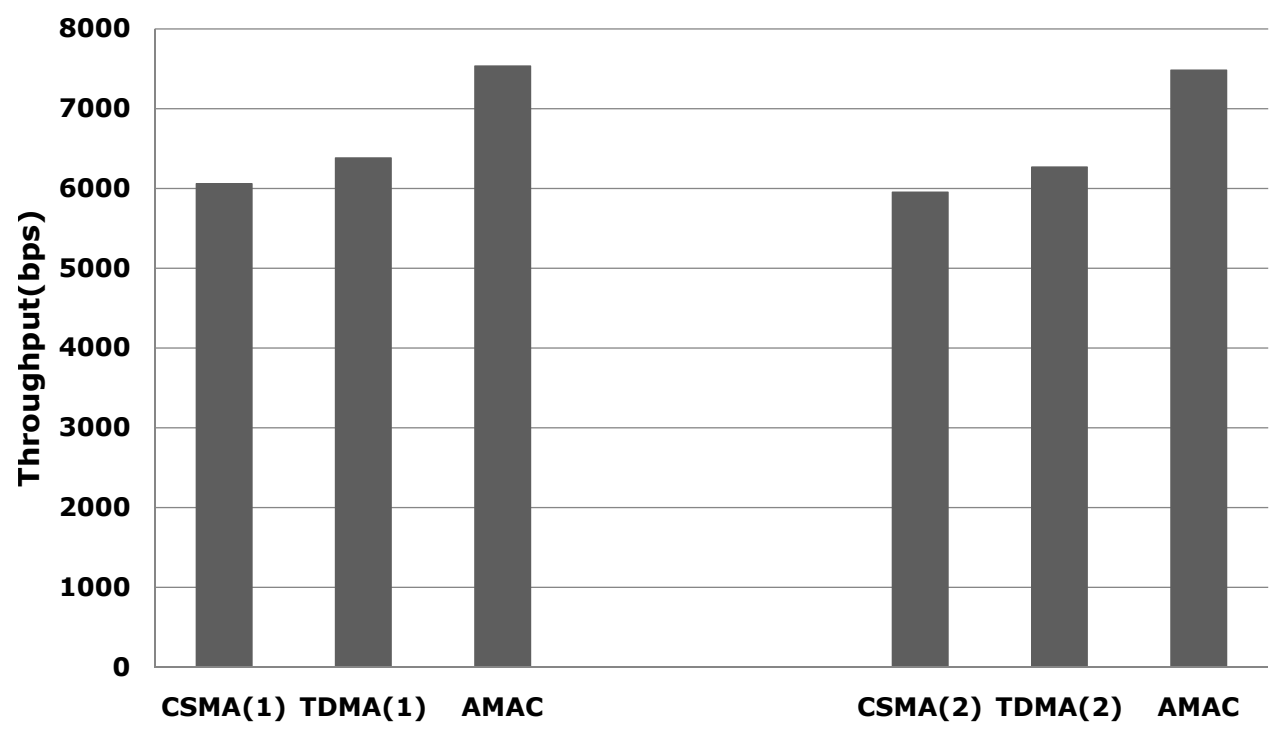

Figure 6.8: Average throughput of CSMA, TDMA and AMAC

\section{(4) Overhead evaluation}

The overhead involved in AMAC from the previous three sets of experiments is calculated in Table 6.2. The payload overhead ratio means the control overhead is divided by the total payload transmitted. Based on the overhead ratio, we can estimate how much GCP bandwidth will be used by the control packets. We compare the payload overhead with different traffics and network conditions. It is observed from the results that the overhead introduced by the MAC switching is relatively small, typically less 
than $1 \%$. Due to the poor radio activity of GNUradio node pair 2, in fix UDP packet size scenario, node pair 1 has to keep requesting switch messages in GCP while performance drops and wait till node pair 2 finishes transmitting 10 packets. Besides, there are more switch requests because of irregular radio activities of GNUradio nodes. That is why node pair 1 has more than twice control overhead compared to node pair 2 . In the mix packet scenario, the switch request messages are initiated only when the average packet size is changing above or below the threshold. Additionally, flow 1 has three nodes which compensate the slow transmission speed of flow 2 so the difference of two flows' radio activities can be ignored. Thus, the overhead ratio is less than $0.1 \%$ for both flows. Of course, this number will increase as the number of nodes in the network increases, but as shown in [4] it is possible to aggregate GCP control packets to prevent exponential increase in network overhead.

\begin{tabular}{|c|c|c|}
\hline Experiment Scenario & $\begin{array}{l}\text { Node Pair } 1 \\
\% \text { control overhead }\end{array}$ & $\begin{array}{l}\text { Node Pair } 2 \\
\% \text { control overhead }\end{array}$ \\
\hline $\begin{array}{l}\text { TCP AMAC } \\
\text { (CSMA) }\end{array}$ & $\begin{array}{l}0.1634 \% \\
(1636 \mathrm{~B})\end{array}$ & $\begin{array}{l}0.184 \% \\
(1840 \mathrm{~B})\end{array}$ \\
\hline $\begin{array}{l}\text { TCP AMAC } \\
\text { (TDMA) }\end{array}$ & $\begin{array}{l}0.2068 \% \\
(2070 \mathrm{~B})\end{array}$ & $\begin{array}{l}0.246 \% \\
(2462 \mathrm{~B})\end{array}$ \\
\hline $\begin{array}{l}\text { UDP AMAC } \\
(25 \mathrm{kbps} \text { load }) \\
(1500 \mathrm{~B} \text { pkt size })\end{array}$ & $\begin{array}{l}0.408 \% \\
(1240 \mathrm{~B})\end{array}$ & $\begin{array}{l}0.1768 \% \\
(521 \mathrm{~B})\end{array}$ \\
\hline $\begin{array}{l}\text { UDP AMAC } \\
(25 \mathrm{kbps} \text { load }) \\
(500 \mathrm{~B} \text { pkt size) }\end{array}$ & $\begin{array}{l}0.255 \% \\
(240 \mathrm{~B})\end{array}$ & $\begin{array}{l}0.111 \% \\
(104 \mathrm{~B})\end{array}$ \\
\hline $\begin{array}{l}\text { UDP AMAC } \\
(25 \mathrm{kbps} \text { load) } \\
\text { (Mix of } 100 \mathrm{~B} \text { and } \\
1500 \mathrm{~B} \text { pkt size) }\end{array}$ & $\begin{array}{l}0.045 \% \\
(255 \mathrm{~B})\end{array}$ & $\begin{array}{l}0.04 \% \\
(160 \mathrm{~B})\end{array}$ \\
\hline
\end{tabular}

Table 6.2: AMAC overhead from GCP control traffic 


\section{Chapter 7}

\section{Conclusion}

In this thesis, AMAC algorithm is presented by using GCP-based control framework in cognitive radio network. Each node has ability to determine when to choose suitable MAC protocol and the network has ability to reach the common MAC protocol based on most nodes' interests. We have experimentally studied the AMAC protocol and selected different scenarios for NS-2 simulation. With different traffic types, AMAC protocol is able to adapt well and reach $20 \%$ more throughput compare to single MAC protocol. In mobile scenario, AMAC is able to preserve specific service requirement and balance the performance of different types network by PHY and MAC adaptation. Our proof-of-concept implementation with GNU radios shows that it is possible to implement dynamic MAC switching in cognitive radios networks using the capabilities of the control plane protocol. In a static environment, Experimental results with a small network show that AMAC is still able to track the better performance of candidate MAC protocols even without PHY adaptation. The results also present MAC switching can provide performance improvements in dynamic application environments, and show that switching latency and control overhead are not excessive. 


\section{References}

[1] J.Mitola. Cognitive Radio: An Integrated Agent Architecture for Software Radio. PhD thesis, Royal Institute of Technology (KTH), 2000.

[2] D. Maldonado, B. Le, A. Hugine, T. W. Rondeau, and C. W. Bostian. Cognitive radio applications to dynamic spectrum allocation: a discussion and an illustrative example. In IEEE DySPAN, pages 597-600, 2005.

[3] Allocation Networks Jun and Jun Zhao. Distributed coordination in dynamic spectrum. In IEEE DySPAN, pages 259-268, 2005.

[4] Xiangpeng Jing and Dipankar Raychaudhuri. Global control plane architecture for cognitive radio networks. In ICC, pages 6466-6470, 2007.

[5] Xiangpeng Jing, Shanmuga S Anandaraman, Mesut Ali Ergin, Ivan Seskar, and Dipankar Raychaudhuri. Distributed coordination schemes for multi-radio coexistence in dense spectrum environments: An experimental study on the orbit testbed. In IEEE DySPAN, pages 597-600, 2008.

[6] D. Raychaudhuri. Orbit: Open-access research testbed for next-generation wireless networks. NSF Network Research Testbeds Program, NSF award ANI-0335244, 2003.

[7] N. Jain, S. Das, and A. Nasipuri. A multichannel csma mac protocol with receiverbased channel selection for multihop wireless networks. In ICCCN 2001, 2001.

[8] Xiangpeng Jing and Dipankar Raychaudhuri. A spectrum etiquette protocol for efficient coordination of radio devices in unlicensed bands. In Proceedings of PIMRC, 2003 .

[9] Carlos Cordeiro and Kiran Challapali. C-mac: A cognitive mac protocol for multichannel wireless networks. In IEEE DySPAN, 2007.

[10] C. Doerr, M. Neufeld, J. Fifield, T. Weingart, D.C. Sicker, and D Grunwald. Multimac - an adaptive mac framework for dynamic radio networking. In IEEE DySPAN, 2005.

[11] Rahul Dhar, Gesly George, Amit Malani, and Peter Steenkiste. Supporting integrated mac and phy software development for the usrp sdr. In IEEE Workshop on Networking Technologies for Software Defined Radio (SDR) Networks, 2006.

[12] George Nychis, Thibaud Hottelier, Zhuochen Yang, Srinivasan Seshan, and Peter Steenkiste. Enabling mac protocol implementations on software-defined radios. In Networked Systems Design and Implementation, 2009. 
[13] K. Mandke, Soon-Hyeok Choi, Gibeom Kim, R. Grant, R. C. Daniels, Wonsoo Kim, Robert W. Heath Jr, and S. Nettles. Early results on hydra: A flexible mac/phy multihop testbed. In the Proc. of the IEEE Vehic. Tech. Conference, 2007.

[14] Minden, G. J. Evans, J. B. Searl, L. Depardo, D. Petty, V. R. Rajbanshi, R. Newman, T. Chen, and G. Weidling. Kuar: A flexible software-defined radio development platform. In IEEE Dyspan 07, 2007. 


\section{Appendix A}

\section{Appendix}

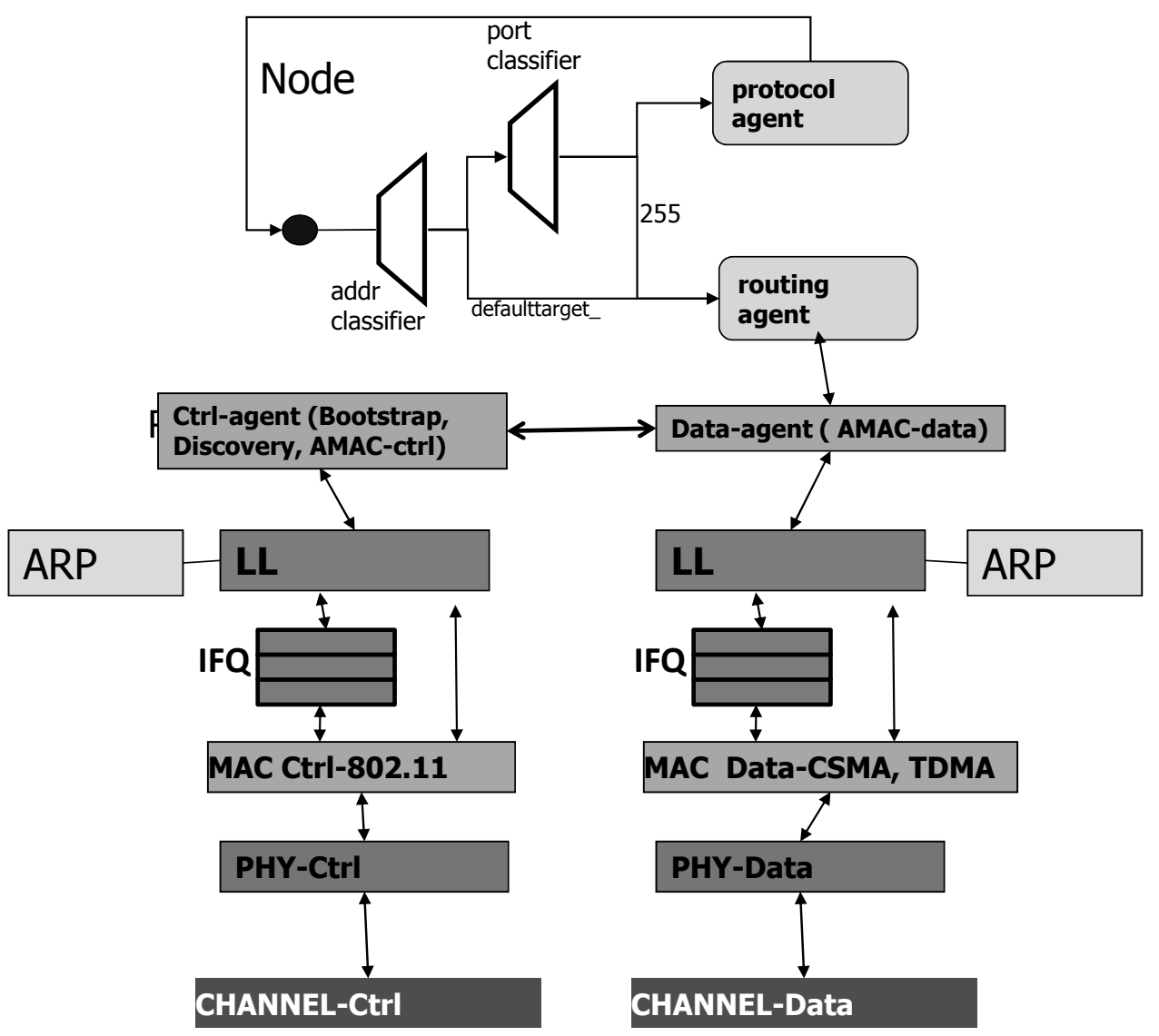

Figure A.1: NS-2 programming flow chart 


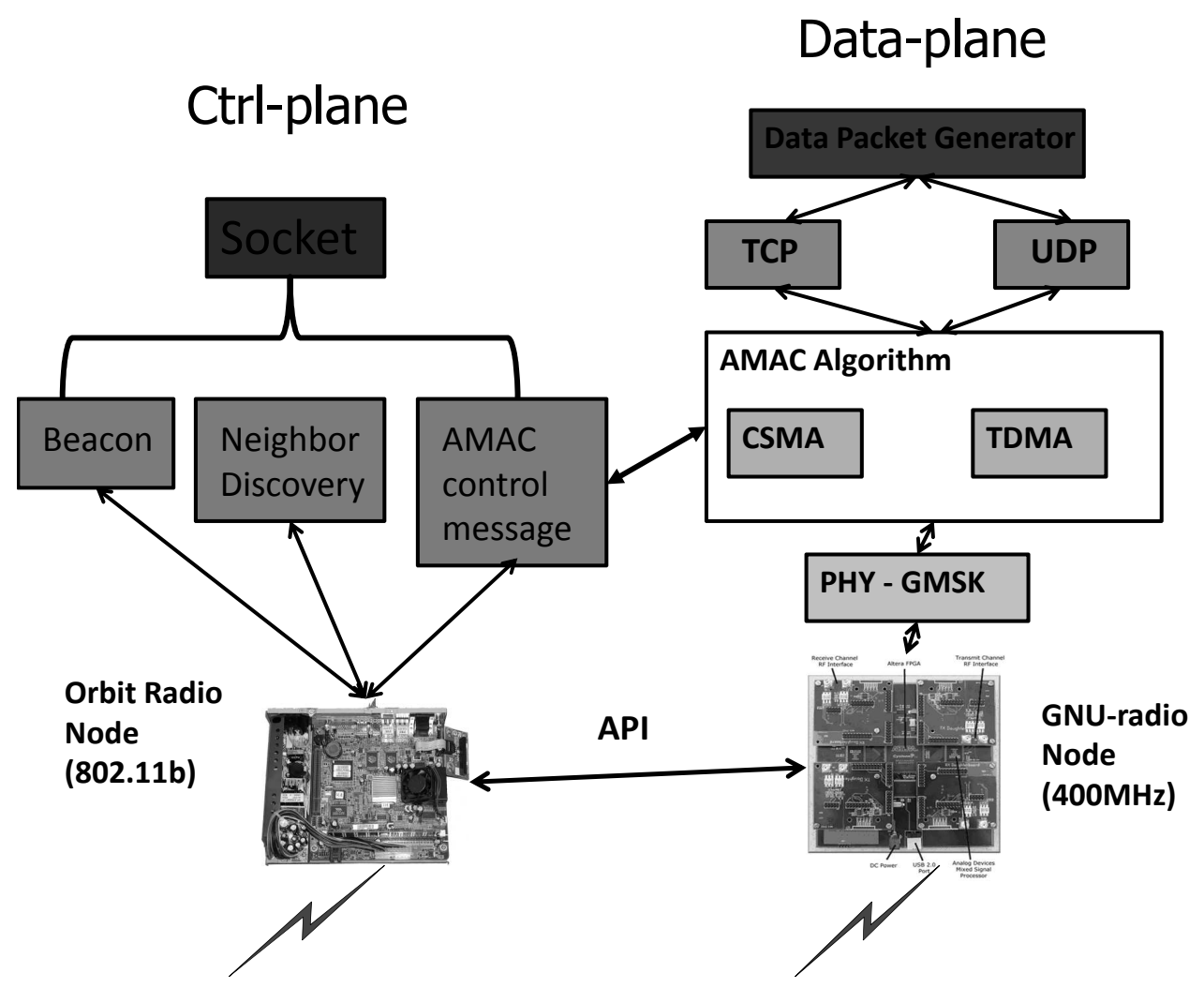

Figure A.2: GNUradio programming stacks 